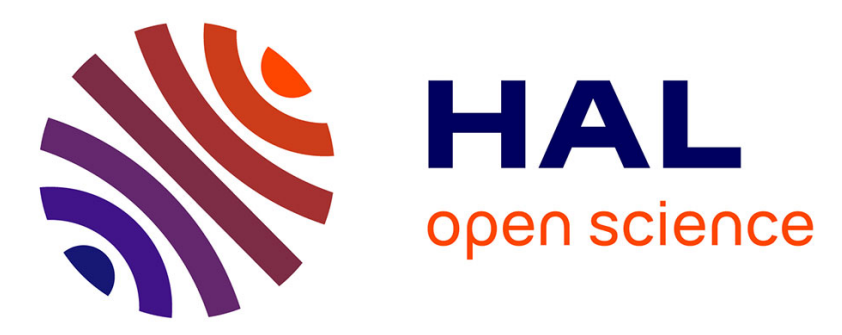

\title{
Propriétés d'un point-mémoire en boracite Fe3B7O 13I ferro-électrique-ferro-élastique orthorhombique
}

\author{
L.A. Pétermann, H. Schmid
}

\section{To cite this version:}

L.A. Pétermann, H. Schmid. Propriétés d'un point-mémoire en boracite Fe3B7O 13I ferroélectrique-ferro-élastique orthorhombique. Revue de Physique Appliquée, 1976, 11 (4), pp.449-466. 10.1051/rphysap:01976001104044900 . jpa-00244080

\section{HAL Id: jpa-00244080 https://hal.science/jpa-00244080}

Submitted on 1 Jan 1976

HAL is a multi-disciplinary open access archive for the deposit and dissemination of scientific research documents, whether they are published or not. The documents may come from teaching and research institutions in France or abroad, or from public or private research centers.
L'archive ouverte pluridisciplinaire HAL, est destinée au dépôt et à la diffusion de documents scientifiques de niveau recherche, publiés ou non, émanant des établissements d'enseignement et de recherche français ou étrangers, des laboratoires publics ou privés. 


\title{
PROPRIÉTÉS D'UN POINT-MÉMOIRE EN BORACITE $\mathrm{Fe}_{3} \mathrm{~B}_{7} \mathrm{O}_{13} \mathrm{I}$ FERRO-ÉLECTRIQUE-FERRO-ÉLASTIQUE ORTHORHOMBIQUE
}

\author{
L. A. PÉTERMANN $\left({ }^{*}\right)$ et H. SCHMID \\ Battelle, Centre de Recherche de Genève, CH-1227 Carouge-Genève, Suisse
}

(Reçu le 3 mars 1975, révisé le 2 février 1976, accepté le 11 février 1976)

Résumé. - Les propriétés ferro-électriques, ferro-élastiques et électro-optiques de la boracite $\mathrm{Fe}_{3} \mathrm{~B}_{7} \mathrm{O}_{13} \mathrm{I}$ orthorhombique permettent d'envisager l'utilisation de ce cristal en tant que pointmémoire électro-optique.

L'étude expérimentale a fourni les principaux résultats suivants :

- La vitesse de propagation de paroi, $v$, des domaines à $180^{\circ}$ est une fonction exponentielle de l'inverse du champ électrique $E, v=v_{\infty} e^{-\delta / E}$. La loi est identique à celle qui a été formulée par Miller et Savage pour le $\mathrm{BaTiO}_{3}$. La vitesse de paroi atteint $10 \mathrm{~ms}^{-1}$ à $92 \mathrm{kV} \mathrm{cm}^{-1}$. A champ électrique constant, la vitesse de paroi varie exponentiellement avec l'inverse de la température absolue ;

- Sous contrainte mécanique extérieure nulle, il n'y a pas de seuil de basculement intrinsèque dans ce matériau, du moins au-dessus de $0,7 \mathrm{kV} \mathrm{cm}^{-1}$;

- Les domaines basculés électriquement dans une matrice à polarisation opposée reprennent leur polarisation initiale par retour élastique spontané sous champ électrique nul ; seules des bandes parallèles de domaines à polarisation antiparallèle sont stables ;

- Un matériau de bonne qualité ne montre encore aucun phénomène de fatigue après plus de $10^{8}$ basculements complets ;

- En diminuant l'angle de cisaillement (par abaissement de la température) le basculement par propagation d'une ou deux parois fait place à un basculement par polynucléation ;

- Les effets de proximité mécanique et électrique ne permettent pas de disposer plusieurs pointsmémoire en échiquier et de les faire fonctionner par accès cross-bar sans interactions mutuelles.

Abstract. - The ferroelectric, ferroelastic and electro-optic properties of orthorhombic boracite crystals $\mathrm{Fe}_{3} \mathrm{~B}_{7} \mathrm{O}_{13} \mathrm{I}$ suggest that this material could be used to design an electro-optic memory element. This study has led to the following main results :

- The velocity of $180^{\circ}$ domain walls is an exponential function of the reciprocal electric field $E: v=v_{\infty} e^{-\delta / E}$. The law is identical to that found by Miller and Savage for $180^{\circ}$ walls of $\mathrm{BaTiO}_{3}$. At constant electric field the wall velocity obeys an exponential function of reciprocal absolute temperature. At room temperature the wall velocity reaches $10 \mathrm{~ms}^{-1}$ at $92 \mathrm{kV} \mathrm{cm}^{-1}$;

- At zero external mechanical constraint, there occurs no electric threshold field, at least down to $0.7 \mathrm{kV} \mathrm{cm}^{-1}$;

- Domains, switched electrically in a matrix of oppositely oriented polarisation, return spontaneously by elastic back-switching at zero electric field ; parallel bands of opposite polarisation are stable after electric switching;

- If the crystal quality is good, the material does not show any fatigue effect, even after more than $10^{8}$ complete polarisation reversals ;

- With decrease of the shearing angle (by decreasing the temperature) the switching mechanism by displacement of one or two walls is replaced by a polydomain nucleation mechanism ;

- Mechanical and electrical proximity effects do not allow an arrangement of several memory elements in checkerboard form on the same monolithic platelet and driving by a cross-bar access without mutual interference. 
1. Introduction. - Les cristaux ferro-électriques, permettant le basculement de l'orientation de l'indicatrice optique lors d'un renversement ou d'une réorientation de la polarisation spontanée, revêtent un intérêt particulier à cause des diverses fonctions d'obturateur optique qu'ils suggèrent : imageur pour ordinateurs optiques, élément d'affichage passif, etc. Les matériaux ferro-électriques de cette catégorie précédemment décrits dans la littérature sont $\mathrm{Bi}_{4} \mathrm{Ti}_{3} \mathrm{O}_{12}$ [1], $\mathrm{Gd}_{2}\left(\mathrm{MoO}_{4}\right)_{3}[2,3], \mathrm{BaTiO}_{3}$ [4] et les pérovskites polycristallins de type PLZT [5, 6]. On peut également exploiter le changement de signe du pouvoir rotatoire, qui intervient pour certaines symétries ferro-électriques lors du renversement de la polarisation spontanée, par exemple dans le $\mathrm{Pb}_{5} \mathrm{Ge}_{3} \mathrm{O}_{11}[7,8,9]$.

En vue d'applications techniques, un matériau de ce genre devrait en particulier réunir les propriétés suivantes :

- Symétrie favorable, permettant une transmission optique optimale, respectivement de 0 et $100 \%$ pour les deux polarisations favorables de domaine entre nicqls croisés, avec compensation de phase pour l'un des deux ;

- Changement nul ou extrêmement petit des composantes du tenseur de déformation spontanée lors de la réorientation du vecteur de polarisation (d'où absence de fatigue mécanique et d'effets de proximité) ;

- Biréfringence ou pouvoir rotatoire élevé (d'où faibles épaisseurs, faibles tensions d'attaque, et compatibilité avec des circuits intégrés de commande);

- Polarisation spontanée et champ coercitif réduits (d'où faible perte d'hystérèse) ;

- Stabilité chimique et thermique ;

- Faibles coefficients piézo-électriques linéaires et quadratiques, garantissant de faibles effets de proximité ;

- Résistivité électrique élevée et uniforme ;

- Faible densité de défauts (d'où mobilité de paroi élevée).

Etant donné que les conditions sont remplies, ou qu'elles peuvent l'être dans une large mesure dans le cas de la boracite $\mathrm{Fe}_{3} \mathrm{~B}_{7} \mathrm{O}_{13} \mathrm{I}$, nous nous sommes efforcés de caractériser la dynamique des basculements de domaines dans des monocristaux de la phase orthorhombique - espèce Aizu [10] $\overline{4} 3 \mathrm{mFmm} 2$ - de ce matériau.

2. Propriétés de la boracite $(\mathrm{Fe}, \mathrm{I})$ et de ses échantillons. - 2.1 PROPRIÉTÉS OPTIQUES ET FERRO-ÉLASTIQUES. - L'orientation de l'indicatrice optique d'un monodomaine de la phase $\mathrm{mm} 2$ de la boracite $(\mathrm{Fe}, \mathrm{I})$ est représentée sur la figure 1 . Elle découle de la corrélation de mesures des biréfringences principales [11] $\left({ }^{1}\right)$,

(1) Dans la référence [12] les biréfringences $\Delta n_{\gamma, \beta}$ et $\Delta n_{\beta, \alpha}$ on été désignées par erreur respectivement $\Delta_{\alpha, \beta}$ et $\Delta_{\gamma, \beta}$.



FIG. 1. - Représentation schématique de l'indicatrice optique d'un monodomaine ferro-électrique/ferro-élastique de la boracite (Fe, I).

des paramètres de la maille [13] et d'expériences de basculements ferro-élastiques [14]. A $29^{\circ} \mathrm{C}$ on trouve [13] :

$a_{\text {o.r. }}=8,65191 \pm 0,000035 \AA / / / n_{\gamma}=1,881_{7}$

$b_{\text {o.r. }}=8,65879 \pm 0,000035 \AA / / / n_{\beta}=1,878_{2}$

$c_{\text {o.r. }}=12,24034 \pm 0,000049 \AA / / n_{\alpha}=1,877_{2} / / P_{\mathrm{S}}$.

Le cristal est donc optiquement biaxial positif puisque $n_{\gamma}-n_{\beta}>n_{\beta}-n_{\alpha}$. Les axes optiques se trouvent dans le plan $n_{\alpha} / n_{\gamma}$. La bissectrice de l'angle aigu est dirigée le long de l'axe de $n_{\gamma}$ avec $2 \mathrm{~V}_{\gamma} \approx 58^{\circ}$, à $20^{\circ} \mathrm{C}$ et $\lambda=546 \mathrm{~nm}$.

Lors du basculement de $180^{\circ}$ de la polarisation spontanée $P_{\mathrm{S}}$, les axes $a$ et $b$ tournent de $90^{\circ}$ et les indices $n_{\gamma}$ et $n_{\beta}$ sont permutés (Fig. 2). Grâce à ce phénomène, on

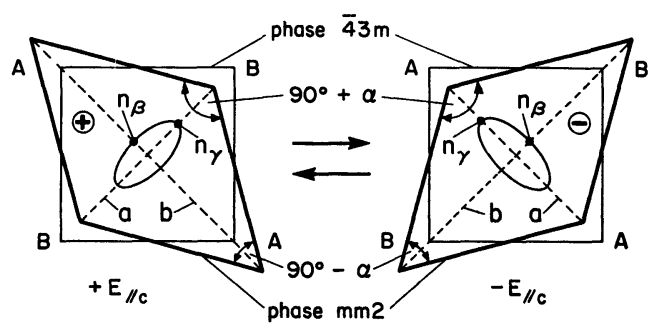

FIG. 2. - Représentation schématique du basculement de l'orientation de la déformation spontanée et de l'indicatrice optique lors du renversement de $P_{\mathrm{S}}$ de $180^{\circ}$ de la boracite $(\mathrm{Fe}, \mathrm{I})$ orthorhomique.

peut obtenir un contraste entre les domaines à polarisation anti-parallèle en observant le cristal en lumière polarisée et avec compensation de biréfringence pour l'un des deux états de domaine ferro-électrique (photos 1 à 3). La biréfringence essentielle responsable de cet effet est la différence $n_{\gamma}-n_{\beta}$, appelée dorénavant $\Delta n_{\gamma, \beta}$. Elle décroît de 0,0045 pour $-70^{\circ} \mathrm{C}$ à 0,003 pour $+70^{\circ} \mathrm{C}$ (transition de phase) [11]. A $20^{\circ} \mathrm{C}$ on mesure 0,004 pour $\lambda=546 \mathrm{~nm}$. L'épaisseur minimale à température ambiante permettant un contraste optimal, est $d \approx 33 \mu \mathrm{m}$, ce qui correspond à une différence 


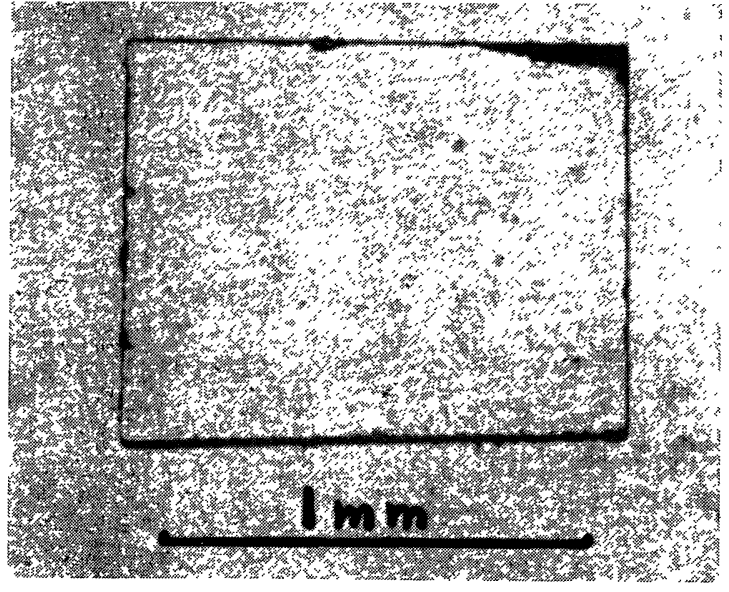

Рното 1.

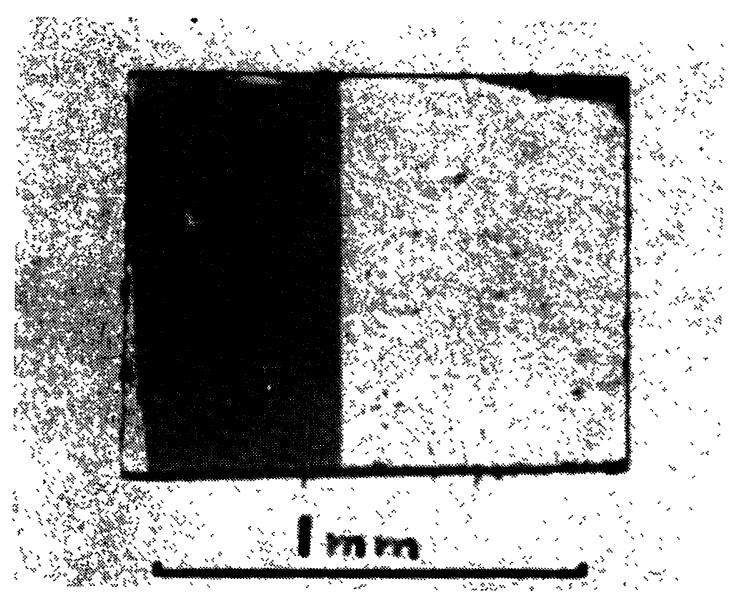

Рното 2.

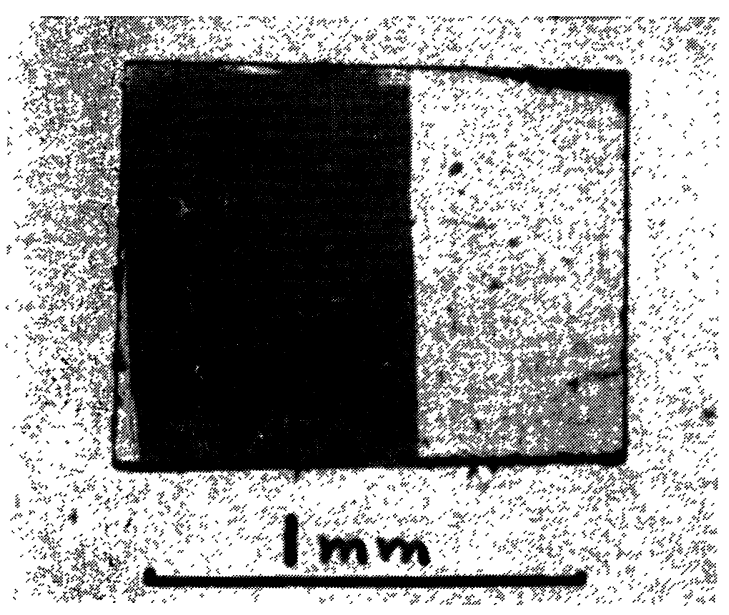

Рното 3.

Pнотоs 1-3. - Basculement par propagation d'une paroi unique, boracite $\mathrm{Fe}$, I (100), épaisseur $d: 96 \mu \mathrm{m}$ (voir Fig. 6). $\mathrm{N}^{\circ} 1$ : Position ouverte. $\mathrm{N}^{\circ} 2$ : Après impulsions de $-43 \mathrm{~V}$ totalisant $50 \mathrm{~ms}$; sens de propagation lent. $\mathrm{N}^{\circ} 3$ : Progression de paroi après nouvelles impulsions de $--43 \mathrm{~V}$, totalisant $20 \mathrm{~ms}$.

de pas optique $\Gamma=\lambda / 2$ pour la plaque cristalline et le compensateur de phase.

Contrairement à la biréfringence et à la polarisation spontanée [18], qui diminuent lorsque la température augmente, la déformation dans le plan $a, b$, caractérisée par l'angle de cisaillement



Fig. 3. - Dépendance de l'angle de cisaillement $\alpha \equiv\left|90^{\circ}-2 \operatorname{arctg} \frac{a}{b}\right|$ avec la température (courbe calculée selon les données de Kobayashi et al. [13] ; domaines d'existence du retour élastique : voir Chapitre 5).

augmente avec la température (Fig. 3). L'angle $\alpha$ est nul à $\sim-60^{\circ} \mathrm{C}$ et atteint $3,2 \mathrm{~min}$ à $70,2^{\circ} \mathrm{C}$. Notons que les angles correspondants d'autres ferro-électriques ferro-élastiques sont bien supérieurs :

$$
\begin{aligned}
& \mathrm{Ln}_{2}\left(\mathrm{MoO}_{4}\right)_{3}(\mathrm{Ln}=\text { Lanthanide }) \quad \sim 10 \quad[15] \\
& \text { PLZT }(x|65| 35) x=0 \% \mathrm{La} \sim 30 \\
& x=6 \% \mathrm{La} \\
& \sim 8\}[16] \\
& \mathrm{BaTiO}_{3} \text { (pour basculements de 90 } 0^{\circ} \text { ) } \quad 30
\end{aligned}
$$

Etant donné le petit angle de cisaillement de la boracite $(\mathrm{Fe}, \mathrm{I})$ on pouvait donc s'attendre à une influence plus faible de cet angle sur le basculement de domaines que ce n'est le cas pour le GMO, par exemple.

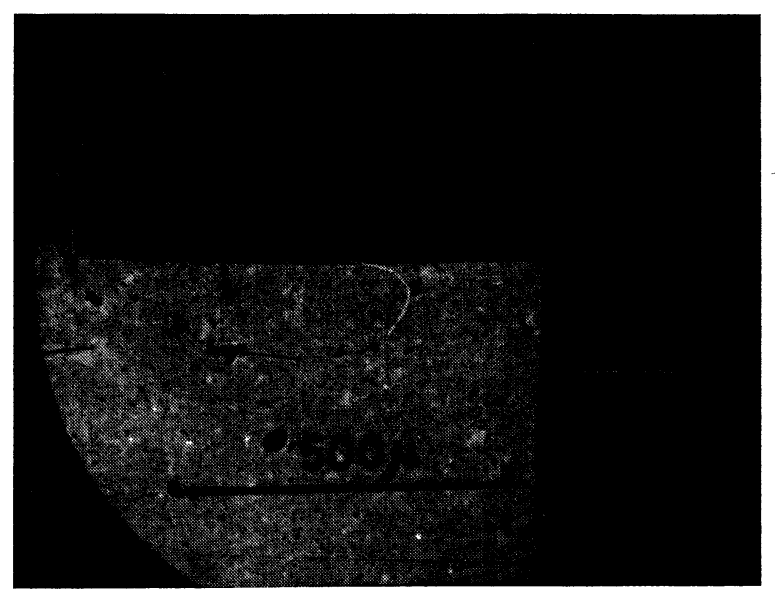

Рното 4. - Blocage de parois orthogonales; épaisseur du cristal $=96 \mu \mathrm{m}$. 


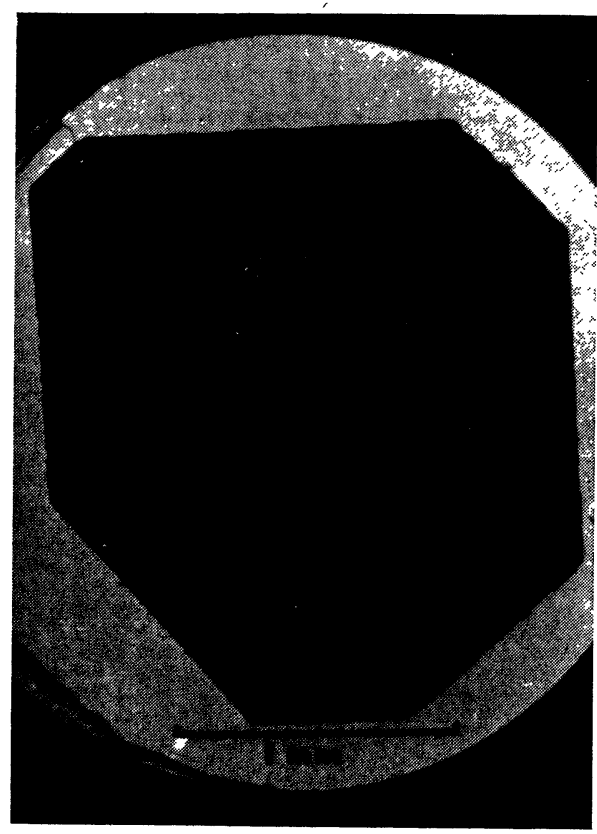

Рното 5.

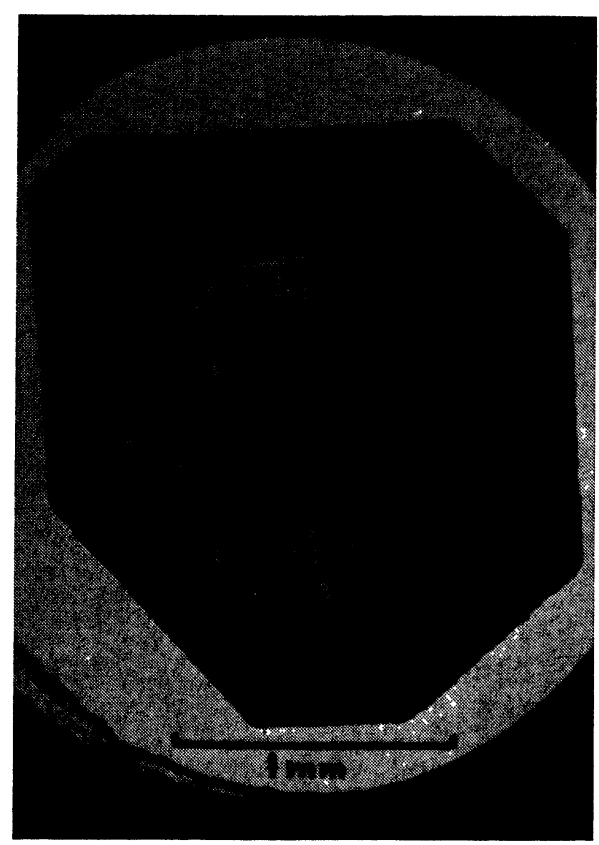

Рното 7.

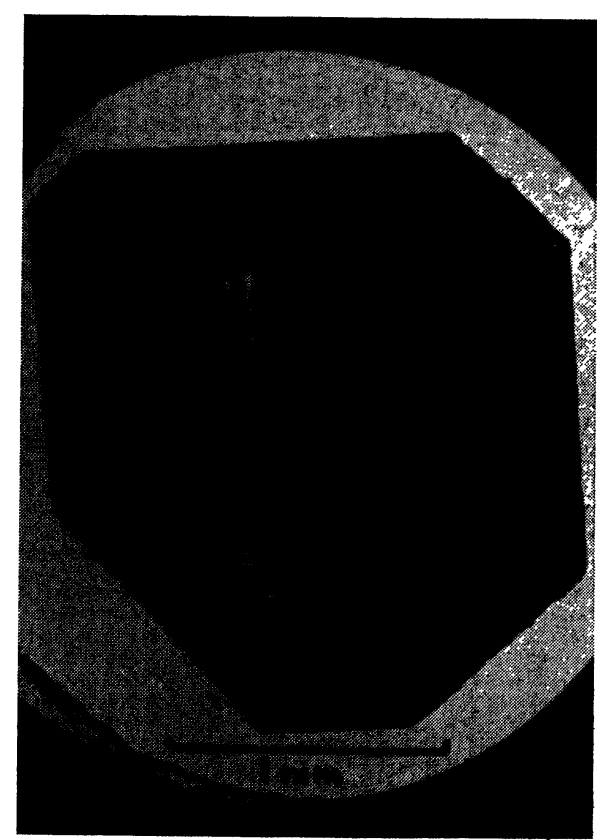

Pното 6.

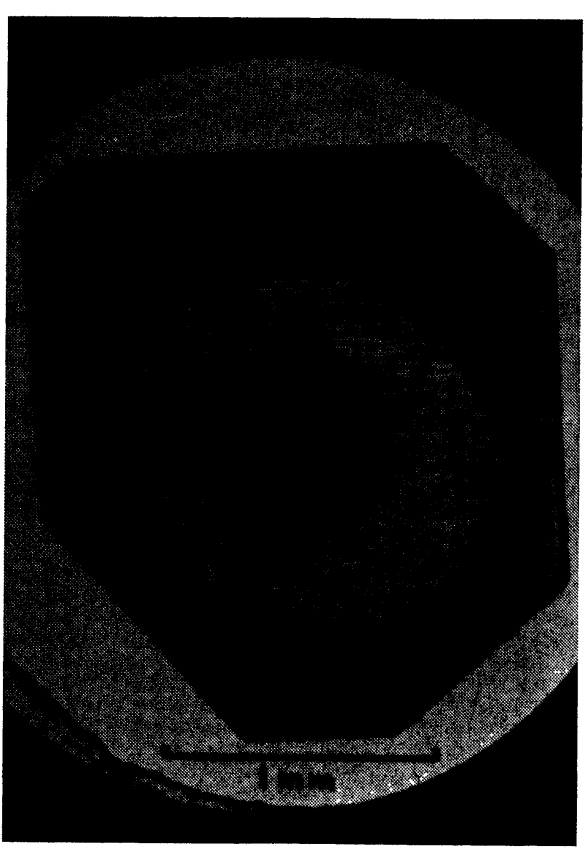

Рното 8.

Pнотоs 5-8. - Basculement lent d'un cristal à électrodes multiples interconnectées; épaisseur $d=53 \mu \mathrm{m}$. No 5 : Etat de saturation; des domaines polarisés dans le plan subsistent sous l'anneau non métallisé. $\mathbf{N}^{\circ} 6:$ Amorce de basculement après 100 impulsions de $-43 \mathrm{~V}, 10 \mathrm{~ms}$; la zone non métallisée n'interrompt pas le domaine baguette. $\mathrm{N}^{\circ} 7$ : Progression irrégulière de paroi, due à 300 nouvelles impulsions $(-43 \mathrm{~V}, 10 \mathrm{~ms})$. $\mathrm{N}^{\circ} 8:$ Idem ; 400 nouvelles impulsions de $100 \mathrm{~ms},-43 \mathrm{~V}$. Le basculement ne progresse plus.

2.2 PropriÉTÉs électriques. - La boracite (Fe, I) mécaniquement libre présente la particularité avantageuse d'un seuil de basculement ferro-électrique très bas (inférieur à $0,7 \mathrm{kV} / \mathrm{cm}$ ) $\left(^{2}\right)$, grâce à laquelle l'étude du basculement peut se faire au moyen de tensions élec-

(2) Voir chapitre 4.2. triques relativement basses, pour des épaisseurs de plaquettes monocristallines assurant encore une bonne résistance mécanique (30 à $100 \mu \mathrm{m})$.

$\mathrm{La}$ résistivité électrique volumique de la boracite (Fe, I) a été mesurée entre 270 et $340 \mathrm{~K}$ sur plusieurs échantillons munis d'électrodes multiples, avec anneau de garde [18]. Cette résistivité est très élevée à tempé- 
rature ambiante $\left(\sim 10^{13} \Omega \mathrm{cm}\right)$, et décroît lorsque la température augmente selon la relation

$$
\rho(T)=1,04 \times 10^{-7} \exp \left(+\frac{13650}{T(\mathrm{~K})}\right) \Omega \mathrm{cm}
$$

pour $270 \mathrm{~K}<T<340 \mathrm{~K}$.

L'énergie d'activation correspondante est de $1,17 \mathrm{eV}$ dans ce domaine de température [18].

La constante diélectrique $\varepsilon$ de cette boracite est environ 11 à température ambiante et varie peu en fonction de la température, si l'on ne s'approche pas trop $\mathrm{du}$ point de transition orthorhombique-cubique ( $349 \mathrm{~K})$.

Ainsi, la constante de temps $R C$ d'un échantillon en forme de plaquette plan-parallèle est

$$
R C=\frac{10^{-11}}{36 . \pi} \cdot \varepsilon \cdot \rho \cong 10 \mathrm{~s}
$$

pour la boracite $(\mathrm{Fe}, \mathrm{I})$ à température ambiante.

Par conséquent, on ne peut pas compter sur le phénomène de décharge spontanée pour annuler rapidement la tension aux bornes d'une telle plaquette, et des effets de proximité électriques sont possibles entre zones voisines d'un même échantillon, avec des temps critiques de l'ordre de 1 à $10 \mathrm{~s}$ (voir chapitre 4.6.1).

\subsection{MÉTallisation Des PlaQuetTES DE BoraCite. -} Le dépôt d'électrodes ( $\mathrm{Au}$ sur $\mathrm{Cr}$ ) a été réalisé par évaporation sous ultravide. Deux configurations d'électrodes ont été réalisées pour nos mesures :

- Anneau de garde circulaire ou rectangulaire, par masquage à l'aide d'un tore de ferrite (photos 5-8) ou d'un élément de grille plane électroformée (photos 2022).

- Quadrillage, par masquage à l'aide d'une grille plane en cuivre ou en nickel (Fig. 8, photos 11-17).

Dans le cas de l'anneau de garde rectangulaire ou de l'électrode quadrillée, il est important d'aligner les axes de la grille sur des axes cristallins cubiques [100] de la plaquette, pour assurer une forme simple et une bonne stabilité de domaines basculés (nous reviendrons sur ce point au chapitre 4.6.3).

2.4 MoNTAGE DES PLAQUeTteS SOUS LE MICROSCOPE. - Les plaquettes munies d'électrodes continues sur les deux faces peuvent être montées simplement entre deux porte-objets recouverts d'une couche conductrice transparente de $\mathrm{SnO}_{2}$. (Voir par exemple photos 1 à 8.)

Le même montage permet aussi d'étudier le basculement ferro-électrique global d'une plaquette munie d'électrodes multiples. Dans ce cas, les diverses zones métallisées d'une même face sont interconnectées par leur contact avec la couche de $\mathrm{SnO}_{2}$ qui les touche.

Par contre, l'étude du basculement partiel d'une plaquette avec électrodes multiples n'est possible que si l'on peut établir le contact séparément pour chaque zone métallisée. A cet effet, on utilise un micro- manipulateur monté sur la platine du microscope polarisant, avec des contacts en fils de platine très fins dont l'extrémité est rendue hémisphérique par fusion locale dans la flamme d'un chalumeau.

2.5 Traitements thermiques. - Une plaquette fraîchement découpée à partir d'un monocristal de boracite contient presque toujours une multiplicité de domaines ferro-électriques. Un traitement thermique - avec contrôle simultané sous le microscope polarisant - permet souvent d'homogénéiser la plaquette :

- chauffage au-dessus du point de Curie,

- refroidissement lent avec ou sans champ électrique appliqué.

3. Equipement de mesure. - Le montage expérimental est schématisé sur la figure 4. Les principaux

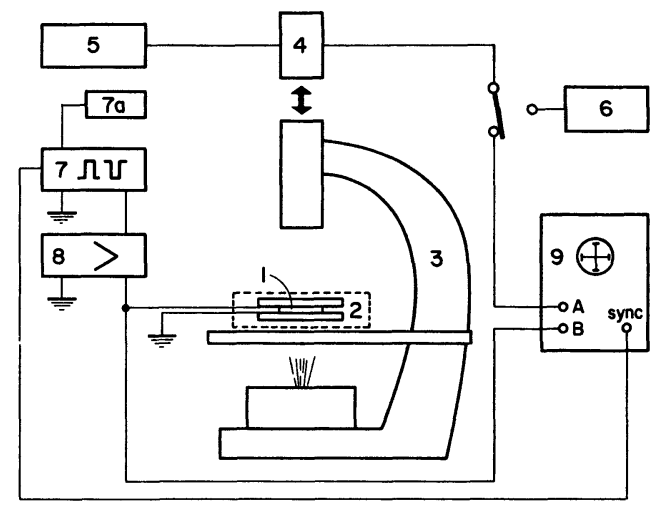

Fig. 4. - Schéma du montage expérimental. - 1. Echantillon et contacts. 2. Four de platine à fenêtres. 3. Microscope polarisant Zeiss GFL. 4. Photomultiplicateur RCA 931A. 5. Alimentation haute tension stabilisée Fluke. 6. Picoampèremètre Keithley 417. 7. Générateur d'impulsions GE 1217-C. 7a. Commande manuelle d'impulsions coup par coup. 8. Amplificateur d'impulsions. 9. Oscilloscope Tektronix 533A.

éléments des systèmes de mesure utilisés sont mentionnés dans la légende de cette figure. En outre, pour les mesures de résistivité électrique et de constante diélectrique, on a recouru à un autre montage comportant essentiellement un micromanipulateur à haute isolation, avec blindage perfectionné, couplé avec un picoampèremètre (Keithley 417) ou avec un capacimètre très sensible (Boonton 72A).

3.1 GÉNÉRATEUR ET AMPLIFICATEUR D’IMPULSIONS. - Les impulsions de tension fournies par le générateur (GE 1217-C) ont une amplitude réglable de zéro à $\pm 43 \mathrm{~V}$, une forme rectangulaire avec un temps de montée et de descente de l'ordre de $0,1 \mu \mathrm{s}$, une durée réglable de $0,1 \mu \mathrm{s}$ à $1 \mathrm{~s}$, une fréquence de répétition réglable de $2 \mathrm{~Hz}$ à $1,2 \mathrm{MHz}$, et une commande manuelle d'impulsions coup par coup.

On utilise deux amplificateurs d'impulsions, tous deux conservant convenablement la forme de l'impulsion originale. Le premier (préamplificateur) permet d'élever le niveau des impulsions jusqu'à $\pm 285 \mathrm{~V}$, et le second jusqu'à $\pm 1,3 \mathrm{kV}$. 
3.2 Dispositif photométrique. - Le temps de commutation d'un élément ferro-électrique peut être déterminé par la mesure du courant associé au renversement de polarisation (voir par exemple méthode Camlibel [19]). Mais cette mesure est globale et ne donne pas d'information précise sur les détails du mécanisme de basculement. En outre, elle est d'autant moins sensible que le basculement est plus lent.

Par contre, la mesure de l'intensité lumineuse traversant le cristal se prête fort bien à l'étude localisée des différentes zones de l'échantillon pendant le basculement. De plus, elle concerne directement le paramètre essentiel du point-mémoire. On utilise à cet effet un photomultiplicateur (PM) monté sur le microscope polarisant.

Pour les mesures de temps de basculement ou de vitesse de propagation de paroi, on branche la sortie de PM sur un oscilloscope. La vitesse de passage d'une paroi limitant deux domaines antiparallèles est déduite de la mesure photométrique, comme l'indiquent les figures $5 a$ et $5 b$ et les photos 18 et 19 .

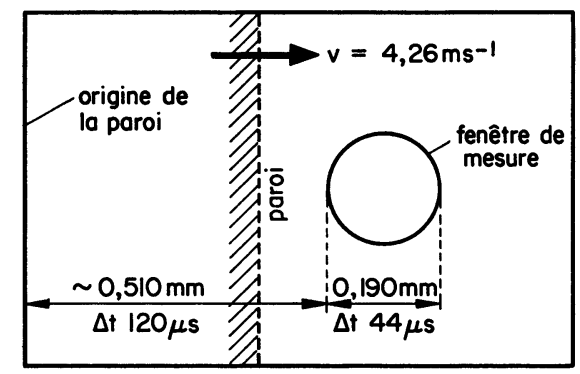

(a)

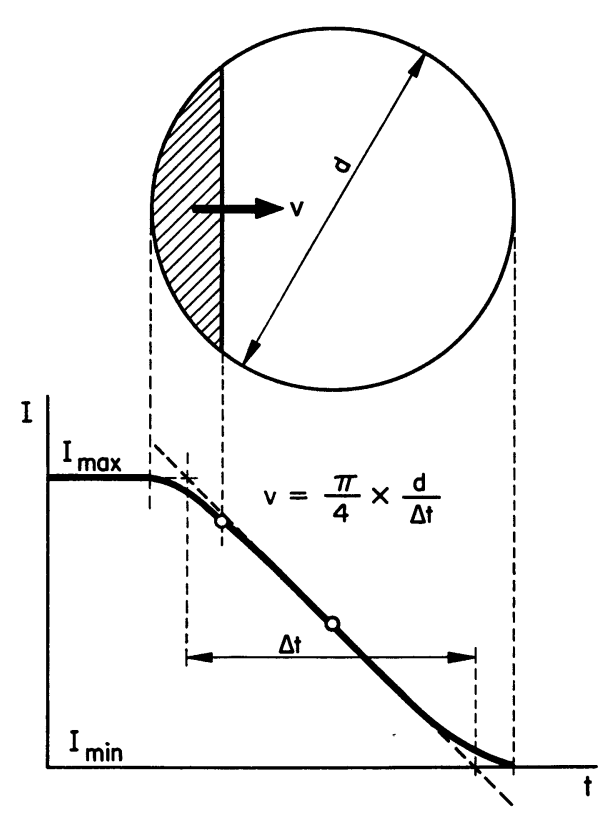

(b)

Fig. 5. - Principe de mesure de la vitesse de paroi par photométrie. a) Interprétation du retard apparent au basculement (voir aussi photo 25). b) Vitesse de paroi déduite de la variation de l'intensité lumineuse à la sortie de l'analyseur du microscope polarisant.
4. Mesures. - 4.1 Transmission et contraste OPTIQUE. - Une plaquette de boracite (Fe, I) (100), d'épaisseur $\leqslant 100 \mu \mathrm{m}$, est presque parfaitement transparente dans le visible [20]. Lorsqu'elle est montée sur le microscope polarisant, le polariseur, le compensateur et l'analyseur étant ajustés pour l'observation optimale du basculement ferro-électrique, le spectre de la lumière transmise en éclairage blanc (couleurs de Newton) dépend fortement de l'épaisseur de la plaquette (diagramme de Michel-Lévy). Le basculement d'un domaine ferro-électrique le fait passer d'un aspect presque noir (biréfringence compensée) à un aspect clair, généralement coloré. Il convient donc de tenir compte de la sensibilité spectrale de la photocathode du PM pour comparer entre elles les valeurs de contraste optique mesurées avec des plaquettes d'épaisseurs différentes.

Le contraste optique entre domaines antiparallèles a été mesuré en utilisant le montage de Sénarmont [21] pour la compensation de biréfringence, avec un éclairage monochromatique et collimaté, $\lambda=546 \mathrm{~nm}$ (filtre interférentiel). La plus grande valeur observée du contraste $\left({ }^{3}\right)$ est 360 .

4.2 Basculement de Domaines. Champ Coercitif. - Pour un échantillon initialement constitué d'un seul domaine (monodomaine) (photos nos 1 à 4), l'amorce du basculement, puis le mouvement d'une paroi située à la limite commune des deux domaines antiparallèles, peuvent se manifester déjà sous un champ électrique inférieur à $1 \mathrm{kV} / \mathrm{cm}$ (comportement semblable du GMO [22]) ; la vitesse de propagation de paroi croît de façon continue avec l'intensité du champ (voir chapitres 4.3.1 et 4.3.2), et la notion de seuil de basculement ne s'impose pas à l'esprit.

Si l'on suit l'avance d'une paroi, sous champ faible et constant, on constate que sa progression n'est régulière que sur de courts trajets, et que la paroi semble rencontrer des obstacles qui ralentissent son mouvement et parfois l'arrêtent complètement. Certains de ces obstacles sont associés à des hétérogénéités visibles du cristal, mais d'autres ne le sont pas. Les obstacles invisibles pourraient être, par exemple, des parois " anti-phase » telles qu'elles ont été observées dans le GMO [23, 24], des limites de secteurs de croissance ou des défauts de polissage. Pour des raisons de symétrie, des domaines anti-phase sont permis dans la phase $\mathrm{mm} 2$ des boracites. De plus, si l'on change le signe du champ faible appliqué sans changer son intensité, on constate une forte asymétrie de la vitesse de propagation (Fig. 6) : il existe une direction de propagation facile pour une polarité donnée, qui est liée au cristal lui-même et non pas à l'asymétrie du montage, ce qu'on établit en retournant le cristal. Cette asymétrie, très prononcée à champ faible, tend à diminuer pour les champs élevés. Un phénomène semblable a été observé par Barkley

(3) Définition du contraste : $K_{\lambda}=$ rapport d'intensités lumineuses transmises. 


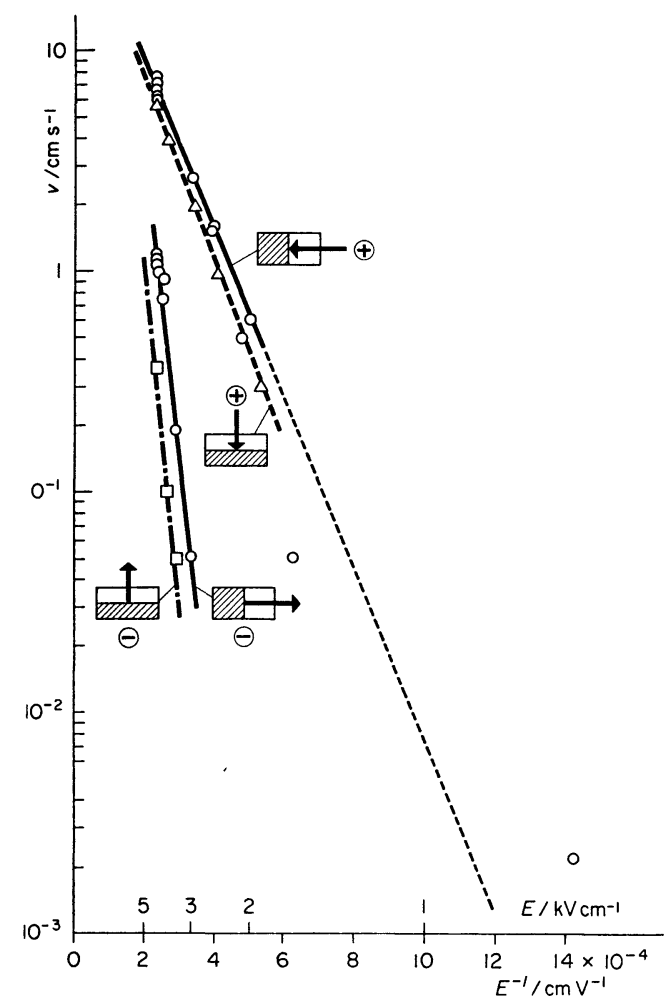

Fig. 6. - Vitesse de propagation de paroi entre domaines antiparallèles en fonction du champ électrique inverse ; déplacement de paroi par impulsion (voir photos 1 à 5); épaisseur de la lame : $96 \mu \mathrm{m}$, surface de la lame : $1,23 \times 0,97 \mathrm{~mm}^{2}$; dissymétrie de la vitesse pour les deux sens de propagation.

et al. [22] dans des plaquettes de GMO (voir discussion, chapitre 6.1).

Si la paroi est complètement arrêtée par un obstacle, la reprise du mouvement peut avoir lieu lorsque l'intensité du champ est augmentée : dans ce cas, les notions de seuil et de champ coercitif acquièrent une signifification claire.

Pour un échantillon du même matériau, mais avec une structure de domaines complexe et irréductible, le basculement ne commence à se manifester que sous un champ électrique de l'ordre de 10 à $30 \mathrm{kV} / \mathrm{cm}$, très supérieur à la valeur citée plus haut. Les parois entre domaines se déplacent à vitesse variable, et la nucléation de nombreux domaines commence à jouer un rôle, d'autant plus important que le champ électrique est plus intense. Les tensions mécaniques locales dues à l'enchevêtrement de domaines de diverses orientations engendrent de véritables seuils de basculement, mais les champs coercitifs correspondants varient d'un endroit à l'autre du cristal. Le phénomène est semblable à celui que décrit Kumada [2] au sujet du molybdate de gadolinium.

Une plaquette monodomaine d'orientation (100), de forme rectangulaire avec bords parallèles aux directions [100] du cristal, bascule pratiquement toujours selon le mécanisme suivant :

- L'un des bords de la plaquette constitue une ligne de nucléation, sur laquelle se forme une paroi à peu près plane qui se propage à travers tout le cristal (photos $\mathrm{n}^{\text {os }} 1$ à 3 );

- Il arrive que la nucléation ne se produise pas partout le long d'un bord, ou qu'elle se fasse simultanément sur plusieurs bords à la fois. Dans ce cas, plusieurs parois se propagent à travers le cristal, toujours perpendiculairement à elles-mêmes et dans des directions parallèles aux axes [100];

- Si deux parois orthogonales se rencontrent, elles peuvent se « bloquer » mutuellement (photo $n^{\circ} 4$ ).

Dans le cas d'une plaquette monodomaine munie d'électrodes multiples, le basculement partiel provoqué par l'application de la tension électrique sur l'une des électrodes s'effectue par déplacement de parois parallèles aux plans (100) du cristal, mais ici les lignes de nucléation ne sont plus seulement constituées par les bords de la plaquette, mais aussi par les limites de l'électrode partielle (photos $n^{\text {os }} 11$ à 17). De plus, on constate l'apparition d'un seuil de basculement bien défini, vraisemblablement induit par les tensions mécaniques (effet ferro-élastique) entre zones voisines de polarisation opposée (analogie avec les résultats obtenus par Kumada [2] sur le GMO).

4.3 Vitesse De propagation De parois. - Deux méthodes de mesure ont été utilisées pour déterminer la vitesse de propagation d'une paroi et sa variation en fonction du champ électrique appliqué. La première, particulièrement bien adaptée aux faibles vitesses, est fondée sur l'observation et la mesure des petits déplacements d'une paroi provoqués par des impulsions de tension rectangulaires, de durée courte et bien connue, commandées manuellement. Nous l'appelons la méthode des impulsions.

La seconde, mieux adaptée aux mesures de grandes vitesses, utilise la variation d'intensité lumineuse transmise à travers une petite zone du cristal (de forme et de dimensions bien connues) provoquée par le passage d'une paroi, dans le montage classique de biréfringence compensée avec l'optique du microscope polarisant. L'intensité lumineuse est mesurée à l'aide d'un PM, dont le courant de sortie est affiché sur l'écran d'un oscilloscope. Nous appelons cette méthode la méthode photométrique.

4.3.1 Méthode des impulsions. - La figure 6 montre les résultats obtenus avec un échantillon sous champ électrique faible $(1$ à $4,3 \mathrm{kV} / \mathrm{cm})$. Les quatre séries de valeurs correspondent aux deux orientations possibles de la paroi unique et aux deux sens de propagation, comme l'indiquent les symboles graphiques associés à chacune des 4 courbes. Le signe représente la polarité du champ appliqué.

On constate une forte dissymétrie de la vitesse de propagation en fonction de la polarité du champ, ainsi qu'une différence systématique de vitesses pour les deux orientations de paroi. La vitesse de propagation n'est pas une fonction linéaire du champ appliqué - 
comme c'est le cas pour le GMO [2] - mais une fonction exponentielle de forme

$$
v=v_{\infty} e^{-\delta / E}
$$

où $v_{\infty}$ représente la vitesse limite pour un champ électrique $E=\infty$ et $\delta$ est un champ d'activation. Une telle relation rend bien compte, sur huit ordres de grandeur, du comportement de la vitesse de parois de $180^{\circ}$ dans le $\mathrm{BaTiO}_{3}$ (Miller et Savage $[25,26]$ ). Hadni etThomas [31] trouvent une relation semblable pour le sulfate de glycocolle (TGS). Dans la théorie de Miller et Weinreich $[27,28]$ élaborée pour le cas de $\mathrm{BaTiO}_{3}$, la forme exponentielle est attribuée à un mécanisme de nucléation de fuseaux de l'épaisseur d'une maille et qui se répandent dans le plan d'une paroi perpendiculairement à la direction de propagation de la paroi. Cette théorie a récemment été mise en question par Baldwin et Milstein [29, 30] qui proposent un mécanisme de tunnelling quantique des ions responsables du basculement ferro-électrique à l'endroit de la paroi.

Le fait que les points de mesure vitesse de paroi $v /$ champ électrique $E$ (voir Fig. 6) s'alignent bien sur des droites en représentation $\log v$-champ électrique inverse, signifie simplement qu'il n'y a pas de seuil défini de basculement.

Dans cette série de mesures, la vitesse la plus petite mesurée est de $2,2 \times 10^{-3} \mathrm{~cm} / \mathrm{s}$ pour un champ de $0,7 \mathrm{kV} / \mathrm{cm}$, la plus grande atteint $7,5 \mathrm{~cm} / \mathrm{s}$ à $4,3 \mathrm{kV} / \mathrm{cm}$.

4.3.2 Méthode photométrique. - Lorsque le diaphragme et le collimateur d'entrée du PM délimitent une fenêtre de mesure circulaire à la surface du cristal, la propagation d'une paroi unique de basculement, à vitesse constante à travers cette fenêtre, fait varier l'intensité lumineuse transmise entre son maximum et son minimum (ou vice versa) selon une fonction du temps qu'il est facile de calculer. La figure $5 b$ représente cette fonction, et montre à partir de quels repères de l'enregistrement photographique on peut calculer la vitesse de propagation de la paroi. Dans le montage utilisé, le diamètre de la fenêtre de mesure photométrique est de $190 \mu \mathrm{m}$ à la surface du cristal.

Lorsque l'on observe par cette méthode le basculement rapide d'un échantillon initialement monodomaine, il est possible de s'assurer que le basculement est bien dû à la propagation d'une paroi unique à travers le cristal, donc de justifier ce procédé de détermination de la vitesse de propagation. A cet effet, on repère la position de la fenêtre de mesure par rapport aux bords du cristal, et on enregistre simultanément à l'oscilloscope, par photographie, la trace de l'impulsion de tension appliquée et celle du signal de sortie du PM. La figure $5 a$ montre que si le basculement a lieu par propagation à vitesse constante d'une paroi issue d'un bord du cristal, la variation d'intensité lumineuse observée à travers la fenêtre de mesure doit commencer à se manifester avec un certain retard par rapport au début de l'impulsion de tension. Le rapport entre ce retard apparent au basculement et la durée de la période pendant laquelle l'intensité lumineuse varie doit être égal au rapport de deux longueurs bien connues : la distance de la fenêtre à l'un des bords, et le diamètre de la fenêtre. La photo $\mathrm{n}^{\circ} 18$ représente le résultat d'une mesure prise dans les conditions particulières illustrées sur la figure $5 a$.

La figure 7 contient les résultats de mesures photométriques de vitesse de paroi dans différents cristaux de boracite $(\mathrm{Fe}, \mathrm{I})(100)$ en fonction du champ électrique appliqué, jusqu'à $85 \mathrm{kV} / \mathrm{cm}$.

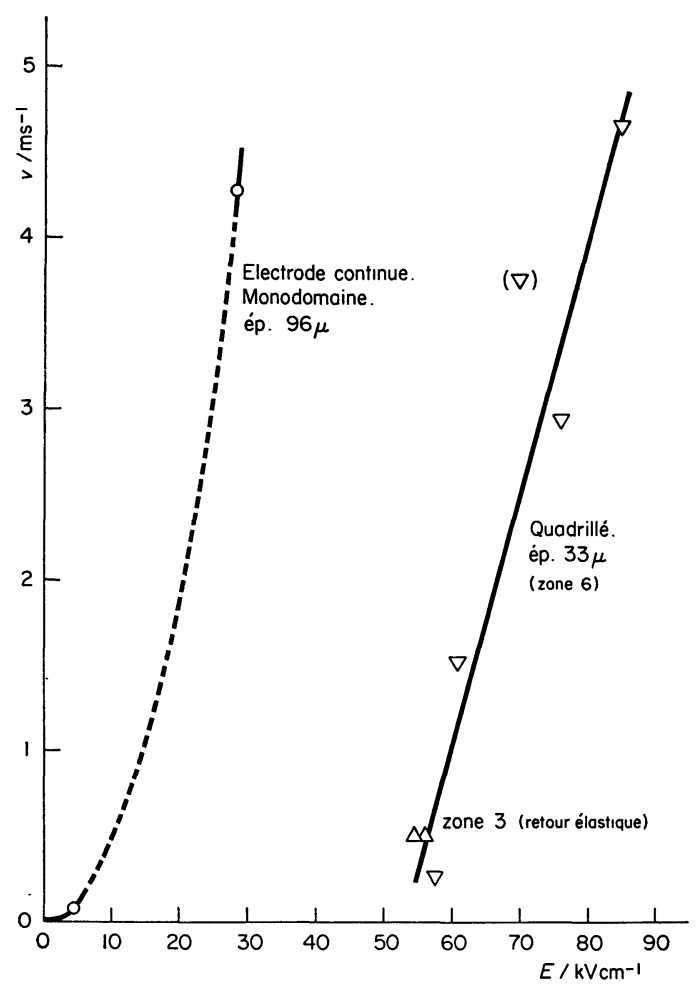

Fig. 7. - Vitesse de paroi avec et sans encadrage du domaine basculant.

La courbe de gauche est obtenue avec le même cristal rectangulaire monodomaine utilisé pour les mesures par la méthode des impulsions, reportées sur la figure 6 ; le petit trait continu $(E<5 \mathrm{kV} / \mathrm{cm})$ représente ces mesures. La méthode photométrique n'a été

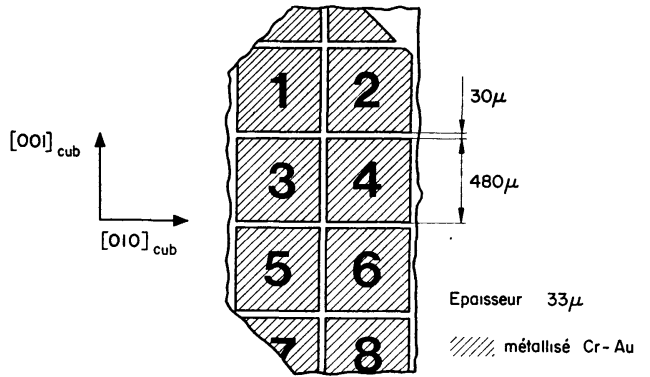

FIG. 8. - Plaquette de boracite (Fe, I) munie d'une électrode quadrillée ; dimensions et numérotation des zones; les bords principaux de la plaquette et les intervalles du quadrillage sont parallèles aux axes [100] cubiques. 


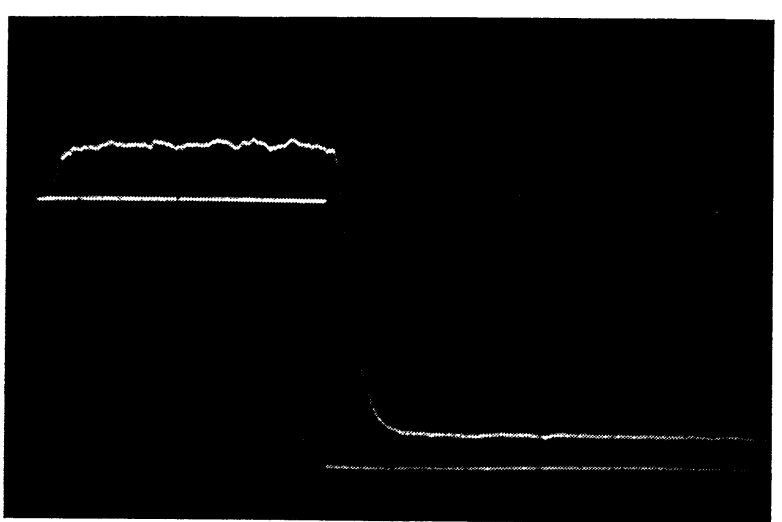

Рното 9.

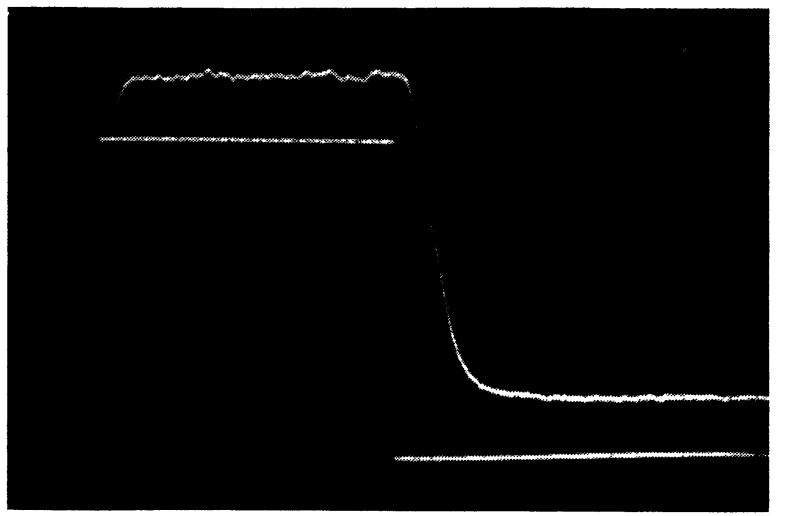

Рното 10.

Pнотоs 9-10 (numérotation des zones : Fig. 8). - Basculement et retour élastique observés par photométrie. $\mathrm{N}^{\circ} 9$ : basculement électrique et retour élastique de la zone 3. Fenêtre de mesure circulaire $190 \mu \mathrm{m}$, échelles d'oscilloscope : $50 \mathrm{~V} / \mathrm{cm} ; 0,2 \mu \mathrm{A} / \mathrm{cm}$; $1 \mathrm{~ms} / \mathrm{cm}$; impulsion : $180 \mathrm{~V}, 4 \mathrm{~ms}$. $\mathrm{N}^{\circ} 10$ : Même zone 3, plus tard ; impulsion : $200 \mathrm{~V}, 4 \mathrm{~ms}$; PM : autre sensibilité ; retour élastique plus lent que la sur photo $\mathrm{n}^{\circ} 9$.

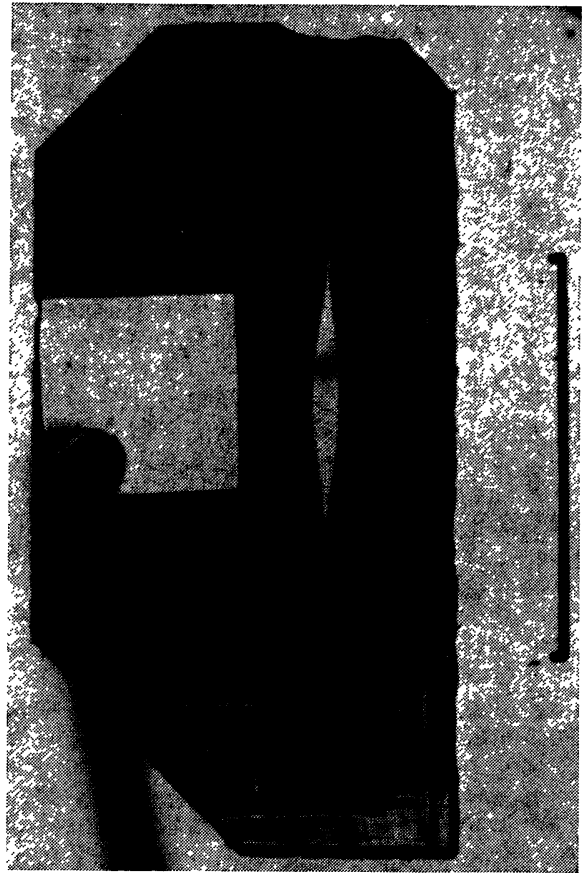

Рното 11

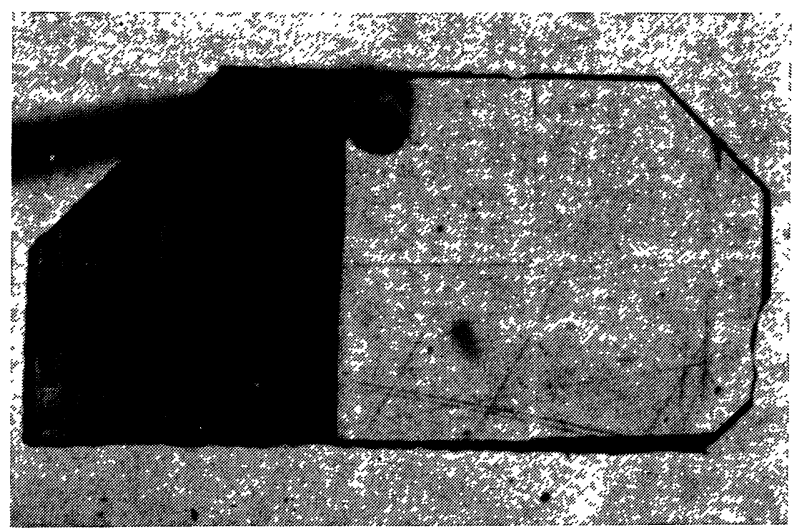

Рното 13.

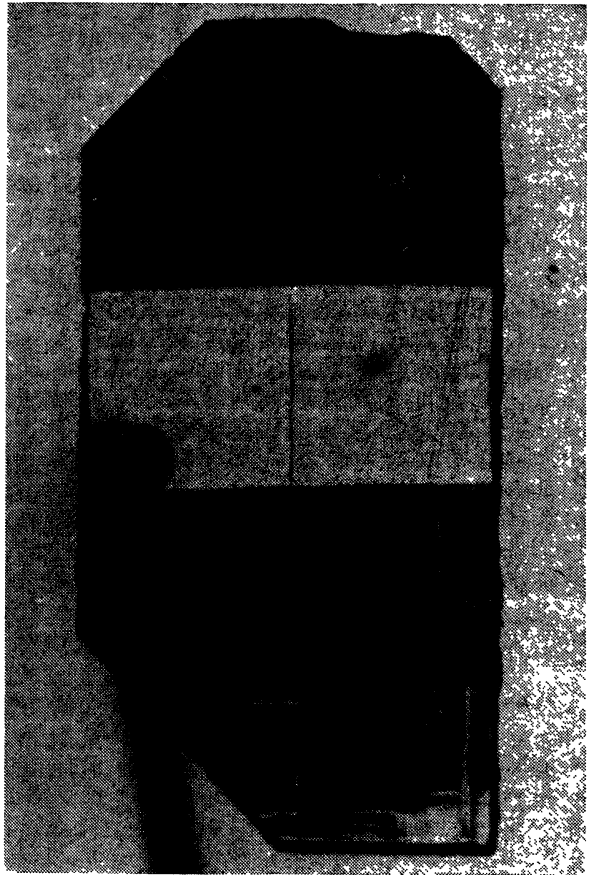

Photo 12

Рнотоs 11-13. - Effets de proximité (électrique et mécanique) sous impulsions électriques de longue durée. $\mathrm{N}^{\circ} 11$ : Impulsion : $145 \mathrm{~V}, 100 \mathrm{~ms}$ sur zone 3. Pas de retour élastique, basculement induit dans la zone 4, fuseau en train de croître. $N^{\circ} 12$ : Contact sur zone 3 , impulsion $145 \mathrm{~V}, \sim 500 \mathrm{~ms}$ fait basculer la zone 4 $\mathrm{N}^{\circ} 13$ : Idem, $145 \mathrm{~V}$ appliqués pendant 1 minute, contact électrique uniquement sur zone 3 , les autres zones sont flottantes. 


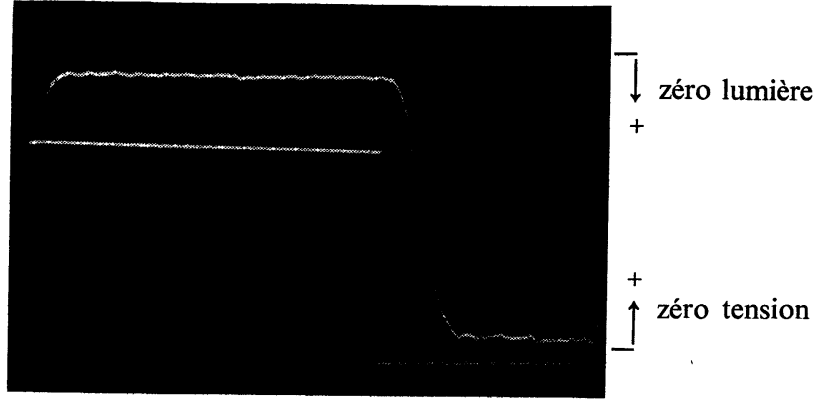

Photo 14.

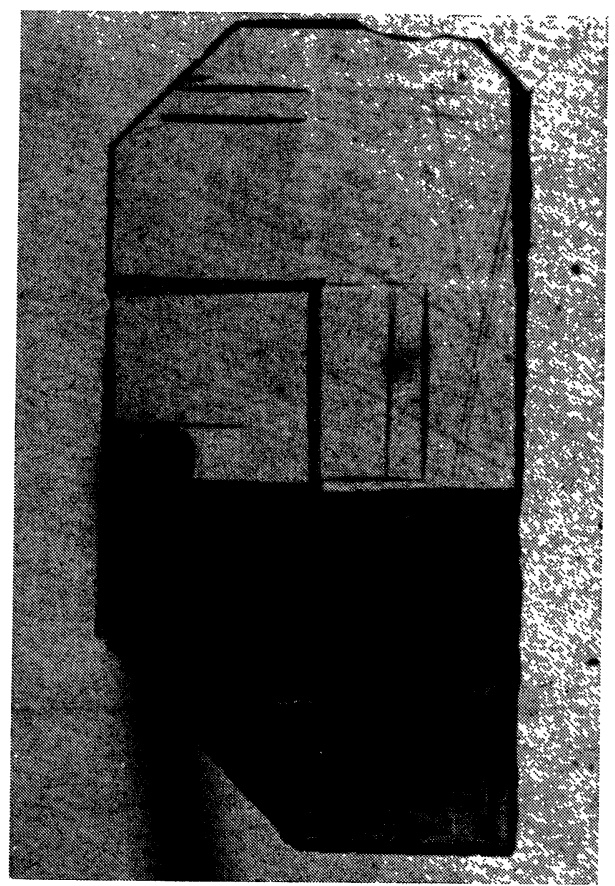

Рното 15.

Рнотоs 14-15. - Bàsculement èt retour élastique d'une zone encadrée (3). $\mathrm{N}^{\mathrm{o}} 14$ : Zone 3, fermeture électrique et ouverture élastique, échelles d'ościlloscope : $0,2 \mu \mathrm{A} / \mathrm{cm} ; 50 \mathrm{~V} / \mathrm{cm}$; $1 \mathrm{~ms} / \mathrm{cm}$; impulsion $185 \mathrm{~V}, 6 \mathrm{~ms}$. No 15 ; Zone 3 encadrée par des domaines antiparallèles, stabilisés sous les intervalles non métallisés. Condition de retour élastique.

utilisée sur cette plaquette que pour une seule valeur de champ (photo $\mathrm{n}^{\circ} 18$ ) où $v$ (paroi) $=4,26 \mathrm{~m} / \mathrm{s}$ pour $E=28 \mathrm{kV} / \mathrm{cm}$, ce qui n'autorise qu'une interpolation approximative représentée par un trait pointillé.

La courbe de droite de la figure 7 concerne le cristal à électrode quadrillée de la figure 8 (voir aussi photos $\mathrm{n}^{\text {os }} 11$ à 17). Deux points de mesure ont été obtenus en faisant basculer la zone 3 du quadrillage, à l'exclusion de son entourage, dans des conditions telles qu'à la fin de l'impulsion électrique, cette zone basculait en sens inverse sous l'effet des contraintes mécaniques dues à son entourage (retour élastique, illustré par les photos $\mathrm{n}^{\mathrm{os}} 9$ et 10 et que nous discutons au chapitre 4.6.2).

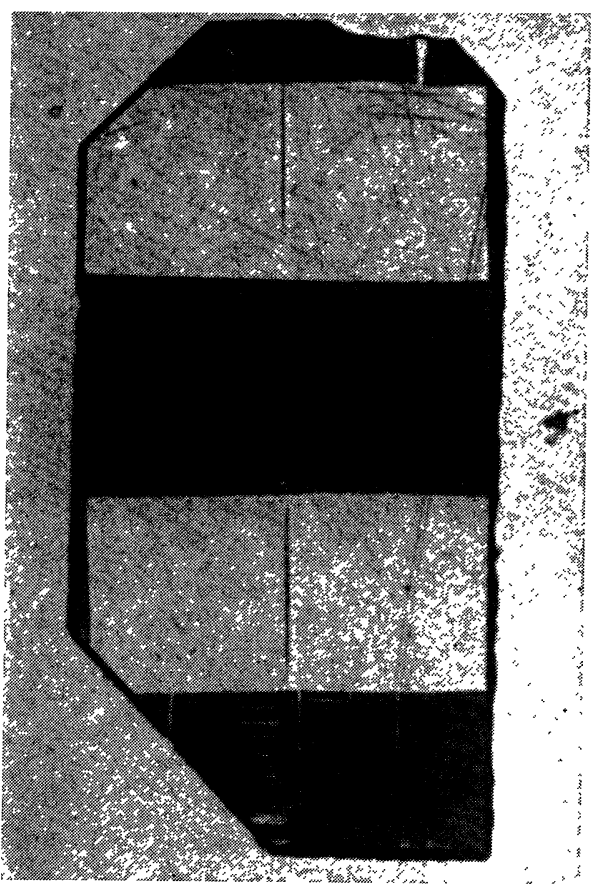

Рното 16.

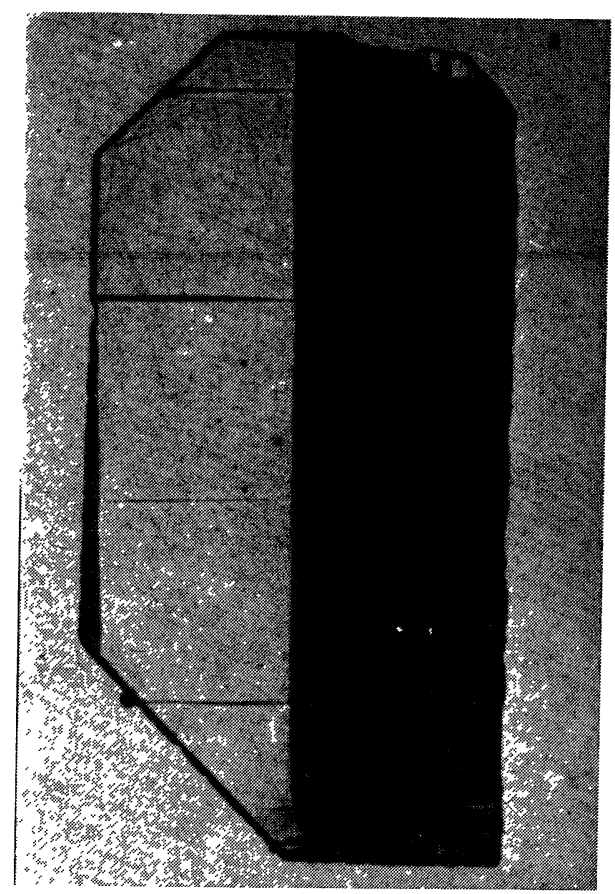

Рното 17.

Pнотоs 16-17. - Configurations de domaines antiparallèles stables (cristal : Fig. 8). Le contact amovible est déplacé d'un carré à l'autre, puis retiré pour les photographies. Impulsions : $300 \mathrm{~V}, 4$ ms. $\mathrm{N}^{\circ} 16:$ Zones $1+2,5+6$ ouvertes. $\mathrm{N}^{\circ} 17$ : Zones impaires ouvertes, basculement obtenu en plusieurs étapes.

La courbe de droite de la figure 7 suggère nettement l'existence d'un seuil vrai et d'un champ coercitif effectif, proche de $50 \mathrm{kV} / \mathrm{cm}$, phénomènes vraisemblablement induits par les tensions mécaniques dues à l'entourage de la zone basculée. 


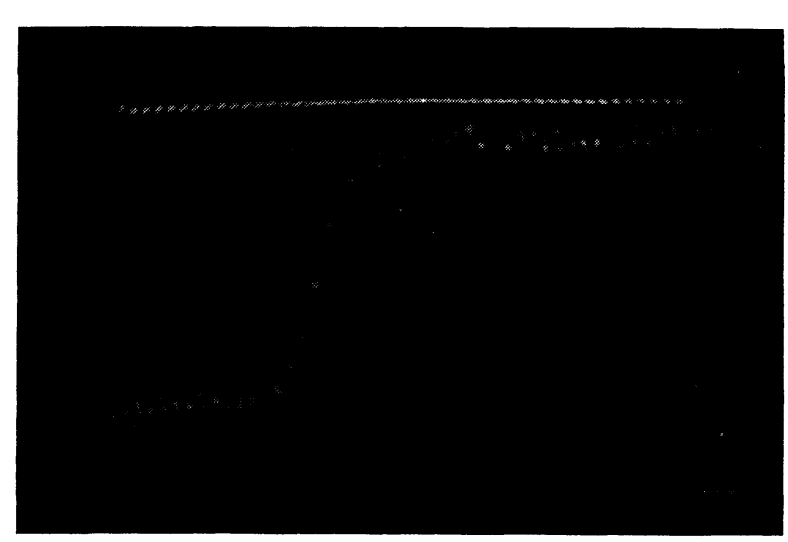

Рното 18.

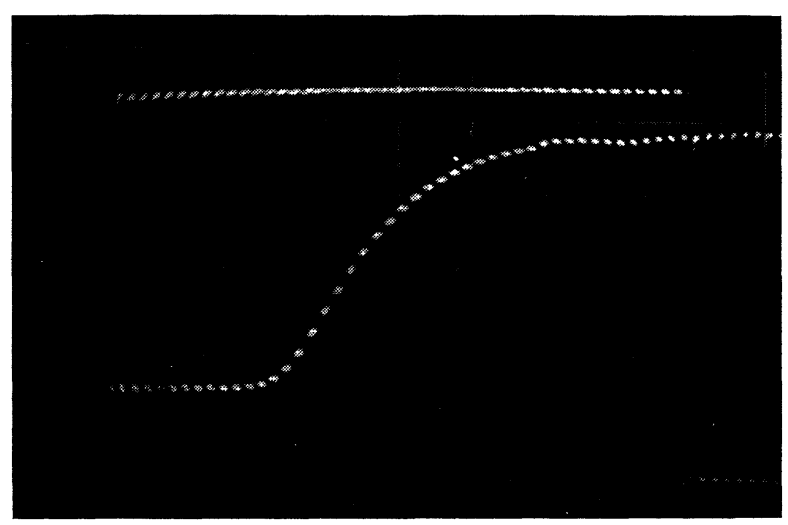

Рното 19.

Pнoros 18-19. - Augmentation de la vitesse de réponse du circuit photométrique (préamplificateur « Teledyne Philbrick 1026FET OP Amplifier »). Voir Fig. 5, disposition de la fenêtre de mesure, et interprétation du retard apparent au basculement. Epaisseur du cristal : $96 \mu \mathrm{m}$. $\mathrm{N}^{\circ}$ 18: (avec amplificateur) $\Delta t=\sim 30 \mu$ s (basculement), échelles d'oscilloscope : $50 \mathrm{~V} / \mathrm{cm}$; $2 \times 10^{-6} \mathrm{~A} / \mathrm{cm} ; 50 \mu \mathrm{s} / \mathrm{cm} ; Z_{\text {in }}$ (ampl.) : $51 \mathrm{k}$; zéros : ligne de base. $\mathrm{N}^{\circ} 19$ : (sans amplificateur), échelles id.; $Z_{\text {in }}$ (oscillo) : $1 \mathrm{M} \Omega, \Delta t$ apjarent $\sim 150 \mu \mathrm{s}$.

Le problème du demi-accès semble aussi résolu dans ces conditions : à $42,5 \mathrm{kV} / \mathrm{cm}$ (demi-champ), la vitesse de propagation de paroi est nulle, ou du moins extrêmement faible.

Mais il subsiste une autre difficulté : si le seuil de basculement est dû aux tensions mécaniques associées au basculement d'un point-mémoire dans le sens de polarisation opposé à celui de son entourage immédiat, le champ coercitif effectif devrait s'annuler, ou même changer de signe (ce qui implique un basculement spontané) lorsque les polarisations d'un point-mémoire et de son entourage sont initialement antiparallèles: C'est effectivement ce que prouve le phénomène de retour élastique qui a été observé.

4.4 Blocage DE PAROIS. - La photo $n^{0} 4$, obtenue au cours des expériences de mesure de la vitesse de propagation d'une paroi sous champ électrique faible, montre une situation de blocage mutuel de deux parois approximativement orthogonales. Au début de l'expé- rience, le cristal rectangulaire monodomaine est entièrement basculé dans le sens correspondant à la transmission optique maximale (position ouvert). Une suite d'impulsions de tension, d'amplitude et de durée constanges, provoque le basculement graduel du cristal. Une première paroi est engendrée tout le long d'un bord du cristal ; un peu plus tard, une seconde paroi se forme le long d'une partie d'un autre bord perpendiculaire au premier. Aussi longtemps que les deux domaines basculés (en position fermé) ne se touchent pas, la propagation de toutes les parois se poursuit par sauts plus ou moins réguliers au rythme de répétition des impulsions électriques. Mais dès que ces deux domaines se touchent, il y a blocage mutuel des deux parois : à chaque nouvelle impulsion de tension, les deux parois orthogonales progressent temporairement pendant la durée de l'impulsion en s'incurvant légèrement, mais leur intersection reste immobile. Pendant l'intervalle entre deux impulsions les deux parois reviennent rapidement à leur position de blocage : c'est ce que nous appelons le blocage élastique. Le phénomène se reproduit indéfiniment (voir chapitre 4.5.2), à moins que la succession des impulsions de tensions mécaniques associées ne finisse par casser le cristal.

$\mathrm{Si}$, à partir d'une situation de blocage élastique, on inverse le signe des impulsions électriques, sans changer leur amplitude ni leur durée, le basculement inverse a lieu par régression des parois, sans blocage.

L'observation de très nombreuses progressions de basculement dans divers cristaux, initialement monodomaines, montre que l'intersection de parois orthogonales ne produit qu'exceptionnellement ce phénomène de blocage élastique. Il est par conséquent possible que ce phénoméne soit lié à l'existence d'une fente ou d'un défaut à l'endroit particulier du cristal où l'intersection des parois a lieu.

Le déblocage, c'est-à-dire la reprise de la progression du basculement à partir d'une situation de blocage élastique, dans le sens même où le blocage est efficace, peut être obtenu de deux manières : soit en augmentant l'amplitude des impulsions, soit en augmentant leur durée, ce qui paraît surprenant au premier abord. La première possibilité suggère que la configuration de blocage élastique engendre un champ coercitif effectif local, d'où l'intérêt considérable de ce phénomène du point de vue du problème du demi-accès. La seconde possibilité implique un comportement non élastique au sein du cristal : ce qu'une suite indéfinie d'impulsions de $10 \mathrm{~ms}$ est incapable de produire, une seule impulsion de 50 ou 100 ms le produit. Il existe un temps de latence pour le basculement dans une telle configuration.

4.5 Phénomène de fatigue. - La durée de vie d'une plaquette de boracite soumise à une succession d'expériences de basculements peut être limitée par divers types de détériorations accidentelles au cours des nombreuses opérations de manutention (découpe, polissage, nettoyages, montages, cycles thermiques, etc.). 
A part ces effets accidentels, donc en principe évitables, aucune fatigue intrinsèque ne devrait en principe exister dans un cristal de bonne qualité. C'est cette absence de fatigue intrinsèque que nous avons surtout essayé de soumettre à l'épreuve par des examens systématiques et des essais de basculement alternés périodiquement pendant de longues durées.

4.5.1 Effets de bord. - Aucune série de basculements répétés dans une plaquette saine n'a entraîné d'amorce de fissure; par contre, dans une plaquette déjà pourvue d'amorces, les basculements répétés provoquent très souvent la propagation des fissures. C'est pourquoi il est très important de tailler les plaquettes avec grand soin, et surtout d'en couper et d'en adoucir les bords sans y créer d'amorces de rupture.

4.5.2 Effet de fatigue particulier d'un cristal à électrodes multiples. - Sur un échantillon muni d'une électrode quadrillée (photo $\mathrm{n}^{\circ} 11$ et Fig. 8) une forme bien particulière de fatigue a été observée affectant la vitesse de basculement inverse d'une zone carrée, sous l'effet des contraintes mécaniques exercées par son entourage, ou vitesse de retour élastique. Lors des premiers essais de basculements, cette vitesse était relativement grande (méthode photométrique : $v>37 \mathrm{~cm} / \mathrm{s}$ photo $n^{0} 9$ ). Déjà après quelques centaines de basculements, cette vitesse avait diminué (photo $\mathrm{n}^{\circ} 10$ ). $\mathrm{Au}$ bout d'un millier d'essais, le retour élastique n'avait plus lieu.

4.5.3 Effets de fatigue de longue durée. - Avec une plaquette de haute qualité et de forme rectangulaire, des basculements complets ont été provoqués à une fréquence de répétition variant entre 250 et $2500 \mathrm{~Hz}$, pendant un total de $50 \mathrm{~h}\left(1,2 \times 10^{8}\right.$ basculements), sans qu'apparaissent les manifestations d'une fatigue intrinsèque du matériau.

Le montage assurait un minimum de contraintes mécaniques grâce à la fixation de contacts permanents en résine conductrice et au choix de fils d'or de $40 \mu \mathrm{m}$ de diamètre, assurant en même temps les contacts électriques et la suspension mécanique de l'échantillon (dimensions : $1,09 \times 0,91 \mathrm{~mm}$; épaisseur $67 \mu \mathrm{m}$ ).

$\mathrm{Au}$ cours de ces essais de longue durée, le basculement du cristal était observé par transmission de lumière (dispositif photométrique décrit au chapitre 3.2), et la forme des impulsions de tension ainsi que le signal PM étaient affichés simultanément sur l'écran d'un oscilloscope (photo $\mathrm{n}^{\circ} 23$ ).

Uniformité du basculement : en déplaçant le cristal sur la platine du microscope, la fenêtre de mesure photométrique peut être placée à n'importe quel endroit du cristal. Cela permet de vérifier que toute la plaquette bascule à chaque demi-période de la tension en créneau, et de déterminer la direction et le mode de propagation des parois entre domaines antiparallèles, grâce à la répartition spatiale du retard au basculement. A $357 \mathrm{~Hz}$, on a constaté que la plaquette basculait par propagation de deux parois symétriques, issues des longs côtés de son périmètre rectangulaire pour l'un des sens de basculement, et du centre de la plaquette pour l'autre sens.

Ce basculement en rideau de théâtre suggère qu'un domaine fusiforme extrêmement mince de polarisation opposée fonctionne comme ligne de nucléation au centre du cristal.

Mesure des caractéristiques du cristal au cours du test de fatigue de longue durée : après $0,56 \times 10^{8}$ et $1,12 \times 10^{8}$ basculements, soit environ au milieu et à la fin du test de fatigue, la plaquette a été observée et photographiée dans ses deux états basculés ouvert et fermé : elle était devenue plus homogène qu'au début des essais, les 45 petits domaines parasites fusiformes dénombrés au début avaient presque tous disparu.

En outre, la vitesse de propagation de paroi a été mesurée dans les deux sens de basculement par la méthode photométrique. Les valeurs obtenues sont indiquées dans le tableau suivant :

\begin{tabular}{|c|c|c|}
\hline $\begin{array}{c}E=25 \mathrm{kV} / \mathrm{cm} \\
(V=170 \mathrm{~V}\end{array}$ & \multicolumn{2}{|c|}{$\begin{array}{c}\text { Vitesse de propagation } \\
\text { de paroi } \mathrm{m} / \mathrm{s}\end{array}$} \\
\hline & ouverture & fermeture \\
\hline Début & 1,66 & 3,74 \\
\hline Milieu $\left(0,56 \times 10^{8}\right.$ basc. $)$ & 4,85 & 6,7 \\
\hline Fin $\left(1,12 \times 10^{8}\right.$ basc. $)$ & 5,4 & 5,0 \\
\hline
\end{tabular}

On constate que la vitesse de propagation de paroi a considérablement augmenté pendant la première moitié du test. De plus, la grande dissymétrie initiale de mobilité en fonction de la polarité du champ a presque complètement disparu à la fin du test.

On peut donc conclure qu'une plaquette de boracite (Fe, I) dans un montage évitant les contraintes mécaniques, ne présente non seulement pas de signes de fatigue intrinsèque après plus de $10^{8}$ basculements, mais au contraire qu'elle s'est sensiblement améliorée. Cet effet peut être comparé au vieillissement bénéfique et stabilisateur de certains résonateurs à quartz piézoélectrique.

4.6 EFFETS DE PROXIMITÉ. - Sous ce titre, nous décrivons les résultats d'expériences obtenus avec des échantillons munis d'électrodes multiples et concernant les influences réciproques entre zones voisines d'une même plaquette monocristalline.

4.6.1 Effets de proximité : retour élastique. - Le phénomène de retour élastique, brièvement mentionné au chapitre 4.3.2, est la manifestation la plus spectaculaire de l'effet de proximité d'origine mécanique. La photo $n^{0} 9$ représente la trace d'une impulsion de tension rectangulaire (amplitude $180 \mathrm{~V}$, durée $4 \mathrm{~ms}$ ) appliquée sur l'électrode de la zone 3 du cristal (Fig. 8), ainsi que la trace de la variation d'intensité lumineuse transmise à travers cette zone. La fenêtre circulaire de mesure photométrique a un diamètre de $190 \mu$, elle est située entièrement à l'intérieur du périmètre de la zone 3 de la plaquette. L'ouverture électrique de cette fenêtre a 
lieu en $300 \mu \mathrm{s}$, au début de l'impulsion de tension, par basculement ferro-électrique, puis la fenêtre reste ouverte jusqu'à la fin de l'impulsion. Dès que le champ électrique est annulé, la fenêtre se referme sans délai visible en $400 \mu \mathrm{s}$, le basculement inverse (retour élastique) étant provoqué par les tensions mécaniques dues à l'entourage antiparallèle de la zone 3 lorsqu'elle est ouverte.

Le retour élastique décrit ci-dessus a été observé quelques centaines de fois, au début du programme de mesures : la vitesse de propagation de paroi diminue avec le nombre d'essais (chapitre 4.5.3.)

Il n'est pas nécessaire que la totalité de l'entourage d'une zone soit polarisée dans un sens unique pour que le retour élastique se produise dans cette zone. Les photos nos 14 et 15 montrent que la zone 3 présente aussi le phénomène de retour élastique, alors qu'elle est entourée de domaines étroits antiparallèles subsistant sous les intervalles du quadrillage démunis de métallisation : lorsque la zone 3 est basculée en position fermée par une impulsion de tension de $6 \mathrm{~ms}$, elle reste fermée jusqu'à la fin de l'impulsion, et sitôt après, le basculement inverse a lieu par retour élastique.

Pour un point-mémoire, ce comportement correspondrait à une remise à zéro automatique.

4.6.2 Effets de proximité : configurations de domaines stables. - Si les effets de proximité sont principalement dus aux tensions mécaniques entre domaines voisins antiparallèles (efforts de cisaillement), il doit exister des configurations de domaines antiparallèles plus stables que d'autres dans une plaquette monocristalline où ces domaines sont astreints à épouser la forme d'électrodes multiples quadrillées.

La stabilité est effectivement intimement liée à certaines symétries de configurations. Les photos $n^{0 \mathrm{~s}} 12$, 13,16 et 17 montrent quelques exemples de répartitions stables de domaines, dans l'échantillon de la figure 8.

La plupart des configurations stables ont en commun une division de la surface en bandes parallèles aux axes principaux du cristal, traversant toute la largeur ou toute la longueur du cristal. Ces configurations stables s'expliquent facilement par les modes de cisaillement de la boracite (voir Fig. 2).

4.6.3 Effets de proximité : angle de cisaillement. Si les effets de proximités dus au cisaillement ne présentent pas un intérêt pratique (par exemple sous forme de remise à zéro automatique par retour élastique), on peut les supprimer en isolant mécaniquement chaque point-mémoire. On peut aussi chercher à diminuer ces effets en agissant sur l'angle de cisaillement du matériau de base (voir chapitre 5).

5. Basculement au-dessous et au-dessus de la température ambiante. - 5.1 MOUVEMENT ET STABILITÉ DE PAROIS EN FONCTION DE LA TEMPÉRATURE. INTRODUCTION. - L'angle de cisaillement dû au basculement ferro-électrique de la boracite $(\mathrm{Fe}, \mathrm{I})$ diminue lorsque la température décroît (voir Fig. 3). Cette propriété particulière de ce matériau a suggéré l'étude de la dynamique de parois en fonction de la température entre $-100^{\circ} \mathrm{C}$ et $+40^{\circ} \mathrm{C}$, dans l'espoir de déceler l'influence de l'angle de cisaillement.

5.2 EChANTILlon. - La plaquette de boracite figurant sur les photos $\mathrm{n}^{\mathrm{os}} 6$ à 12 a été modifiée pour ces essais : les électrodes initiales ont été supprimées, et une nouvelle couche mince métallique semi-transparente a été déposée, uniforme et continue sur une face, en configuration anneau de garde carré sur l'autre face (face naturelle du cristal d'origine). Les arêtes de l'électrode carrée sont soigneusement alignées sur les axes [100] cubiques du cristal. L'épaisseur finale de cette plaquette était de $53 \mu \mathrm{m}$ (photos nos 20 à 22).

5.3 CONTACTS ÉLECTRIQUES. - L'échantillon est muni de trois électrodes seulement. L'électrode continue est connectée par la couche de $\mathrm{SnO}_{2}$ sur laquelle la plaquette repose ; sur l'autre face, le carré métallisé et l'électrode continue qui l'entoure sont chacun muni d'un plot de contact en résine conductrice, sur lesquels viennent s'appuyer deux fils de platine.

5.4 Montage DANS LE DEWAR. - La plaquette monocristalline est montée à l'intérieur d'un petit dewar de bonne qualité optique adapté au microscope polarisant. Sous flux d'azote refroidi à l'air liquide, la température de l'équipage peut être abaissée jusqu'à environ $140 \mathrm{~K}$. La température d'équilibre est ajustée par variation du flux d'azote froid.

5.5 EFFET DE PROXIMItÉ ÉleCTRIQUe. - Lorsque les électrodes de la base et de la zone carrée centrale de la plaquette sont branchées sur le générateur d'impulsions, l'anneau de garde étant flottant, le basculement ferro-électrique peut se propager graduellement à toute la plaquette pour un nombre suffisant d'impulsions de même polarité. Il s'agit certainement d'un effet de proximité électrique, puisque l'on peut le supprimer complètement en connectant l'anneau de garde au potentiel de la base du cristal.

5.6 ENCADRAGES. - A partir d'un état de polarisation uniforme dans toute la plaquette (mono-domaine), une impulsion de basculement est appliquée sur l'électrode carrée centrale seule, l'anneau de garde et la base du cristal étant tous deux à la masse. La zone située sous l'électrode carrée bascule, puis revient rapidement à l'état de polarisation initial lorsque l'impulsion de tension est terminée : c'est le retour élastique que nous avons décrit au chapitre 4.6.2. Il reste cependant une zone étroite basculée tout autour du carré central, sous une partie de l'intervalle non métallisé : sa polarisation étant antiparallèle à celle du reste de la plaquette, nous l'appelons encadrage antiparallèle étroit. Il est bien visible sur la photo $\mathrm{n}^{\circ} 20$, et il se révèle indéfiniment stable en régime d'impulsions brèves.

Si l'expérience est répétée en inversant la polarisation initiale de la plaquette tout entière, et en inversant aussi 


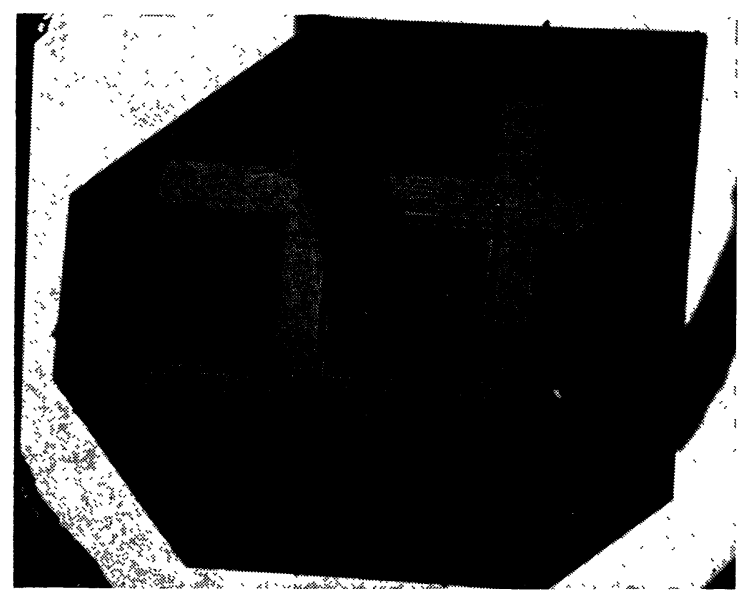

Рното 20.

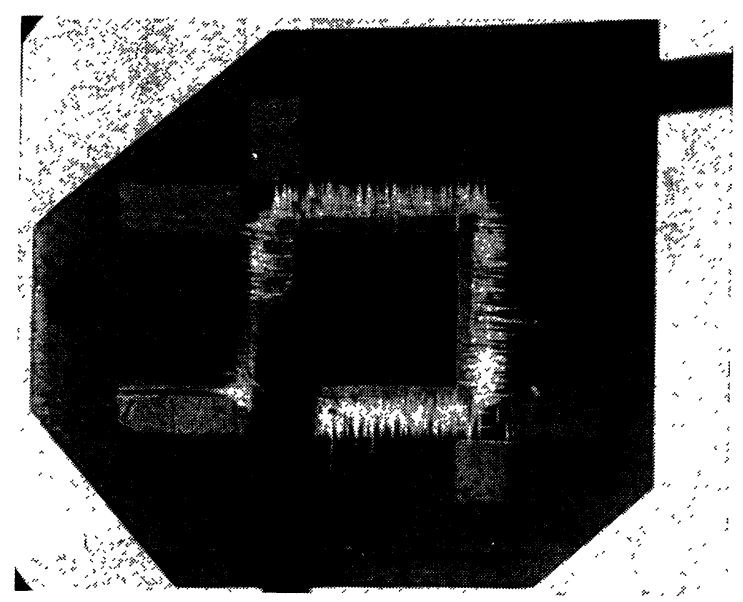

Рното 21.

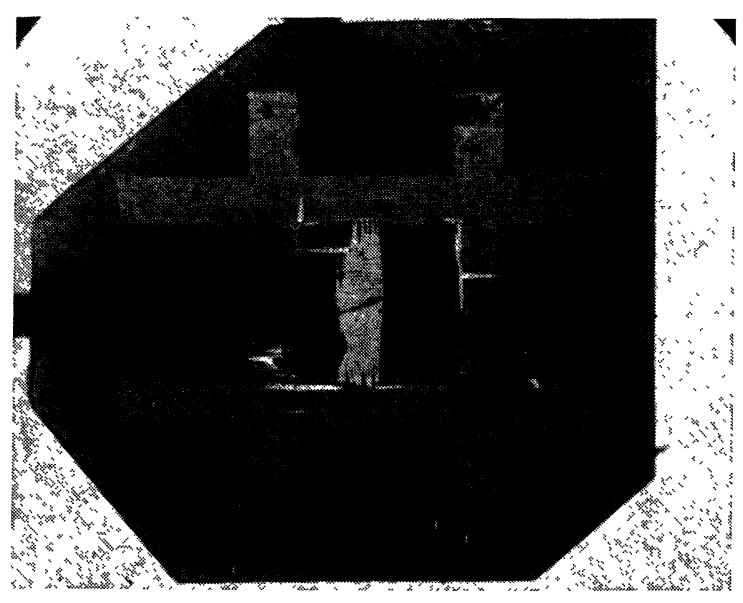

Рното 22.

Pнотоs 20-22. - Basculement d'un carré, plaquette ( $\mathrm{Fe}, \mathrm{I}) 100$, bords du carré parallèles à [100] cubique, épaisseur finale du cristal : $53 \mu \mathrm{m}$; carré central : $476 \times 476 \mu \mathrm{m}^{2}$, intervalle non métallisé : $159 \mu \mathrm{m} . \mathrm{N}^{\circ} 20$ : Encadrage antiparallèle étroit, après 500 basculements du carré central (fermeture) suivis chaque fois du retour élastique (ouverture). $\mathrm{N}^{\circ} 21$ : Encadrage mixte large, centre stable en basculement fermé (noir). $\mathrm{N}^{\circ} 22$ : Domaines polarisés dans le plan, apparaissant dans le carré central après de nombreux basculements sous basse tension (1 400 basculements + retours élastiques, $100 \mathrm{~V}$ ).

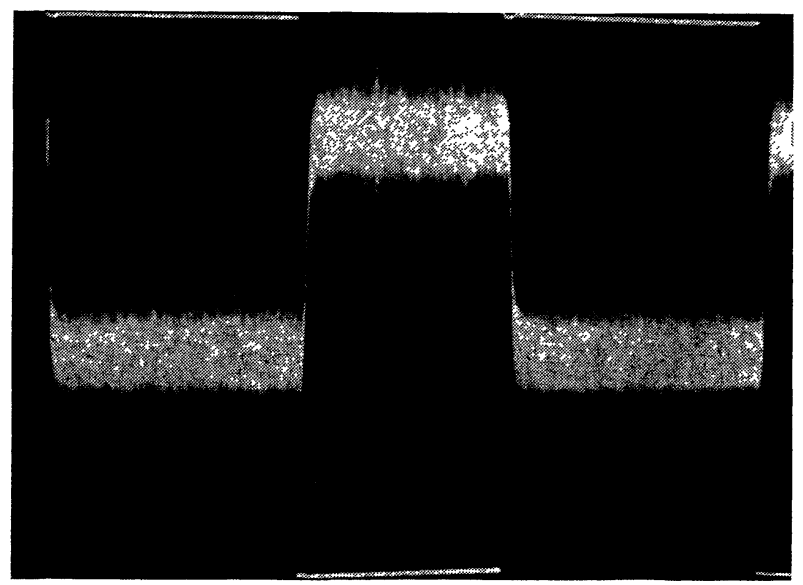

Рното 23. - Basculements alternatifs à $364 \mathrm{~Hz}$. Epaisseur du cristal : $67 \mu \mathrm{m}$. Signaux après $1,1 \times 10^{8}$ basculements. Tension symétrique $\pm 165 \mathrm{~V}$, zéro au centre, $50 \mathrm{~V} / \mathrm{cm}$; lumière transmise : zéro sur la ligne de base. Balayage : $50 \mu \mathrm{s} / \mathrm{cm}$.

le signe de l'impulsion de basculement, on observe également le basculement et le retour élastique du carré central, mais par contre aucun encadrage ne subsiste. Cette asymétrie est probablement due aux tensions mécaniques résiduelles asymétriques qui sont créées lors de la croissance du cristal, de son refroidissement et des opérations de découpe et de polissage d'une plaquette.

On peut également provoquer la formation d'un autre type d'encadrage de domaines en appliquant la tension de basculement pendant une dizaine de secondes sur le carré central de la plaquette : la zone basculée s'étend à toute la région non métallisée située entre les électrodes, et la polarisation s'oriente en partie dans le plan de la plaquette sous l'effet du champ tangentiel créé entre l'électrode centrale et l'anneau de garde. Nous appelons ce deuxième type d'encadrage l'encadrage mixte large, que représente la photo $\mathrm{n}^{\circ} 21$, où l'on reconnaît facilement les domaines noirs et gris antiparallèles, et les franges claires polarisées parallèlement au plan de la plaquette.

Un tel encadrage ne modifie pas sensiblement la vitesse de basculement électrique, mais diminue systématiquement la vitesse du retour élastique, ou même supprime ce dernier : cet effet pourrait être dû à la présence des domaines polarisés parallèlement au plan qui réduirait les tensions mécaniques associées à l'entourage antiparallèle du carré central.

5.7 Mesures de CONTROLE A TEMPÉRATURE AMBiante. - Au début de l'expérience, une série de mesures de vitesse de paroi en fonction du champ électrique a été effectuée par photométrie sur deux plaquettes, la première décrite au paragraphe 5.2 , et la seconde, d'épaisseur $80 \mu \mathrm{m}$, munie d'électrodes semblables. Ces mesures comparatives ont été faites à température ambiante seulement $\left(24^{\circ} \mathrm{C}\right)$; le tableau cidessous en résume les résultats essentiels : 


\begin{tabular}{ccc} 
& \multicolumn{2}{c}{ Plaquettes (Fe, I) $(100)$} \\
Epaisseur & $\mathrm{n}^{\circ} 1$ & $\mathrm{n}^{\circ} 2$ \\
- & $53 \mu \mathrm{m}$ & $80 \mu \mathrm{m}$ \\
Basculement électrique & - & - \\
- vitesse de paroi max. & $1,75 \mathrm{~m} / \mathrm{s}$ & $6,0 \mathrm{~m} / \mathrm{s}$ \\
- champ électrique max. & $41,5 \mathrm{kV} / \mathrm{cm}$ & $33,7 \mathrm{kV} / \mathrm{cm}$ \\
- seuil de basculement & $<10 \mathrm{kV} / \mathrm{cm}$ & - \\
$\begin{array}{c}\text { Retour élastique } \\
\text { - vitesse de paroi max. }\end{array}$ & $0,15 \mathrm{~m} / \mathrm{s}$ & 0,25 à $0,4 \mathrm{~m} / \mathrm{s}$ \\
$\begin{array}{c}\text { (encadrage étroit) } \\
\text { vitesse de paroi max. } \\
\text { (encadrage mixte large) }\end{array}$ & $0,05 \mathrm{~m} / \mathrm{s}$ & \\
& &
\end{tabular}

5.8 MeSuReS A BaSSE TempératURe. - La première plaquette a été refroidie progressivement jusqu'à $170 \mathrm{~K}$, puis réchauffée lentement jusqu'à température ambiante, à plusieurs reprises.

Deux procédés de mesure de la vitesse de paroi ont été utilisés, selon que le retour élastique se produisait ou non :

- Avec retour élastique, le cristal était soumis à une suite d'impulsions de tension fixe, mais de durée variable. Le dispositif de mesure photométrique était utilisé (voir chapitre 3.2). La fenêtre de mesure, circulaire et de diamètre $190 \mu \mathrm{m}$, était placée dans l'un des angles et tangente à deux côtés du carré central ; on mesurait la durée minimale d'impulsion capable de provoquer l'obturation complète de la fenêtre, observée à l'oscilloscope. En répétant la mesure pour différentes amplitudes d'impulsions, on en tirait la vitesse de paroi en fonction du champ électrique appliqué, pour chaque température d'essai, ainsi que la vitesse de paroi lors du retour élastique. Ce procédé n'est plus applicable lorsque la température est inférieure à environ $10^{\circ} \mathrm{C}$, d'une part parce que le retour élastique cesse de se produire, et d'autre part parce que le mécanisme de basculement électrique se modifie graduellement lorsque la température baisse : au-dessous de $10^{\circ} \mathrm{C}$, le basculement n'a plus lieu par propagation d'une paroi unique (ou de deux parois symétriques), mais principalement par nucléation de nombreux domaines approximativement rectangulaires.

- Sans retour élastique, l'observation directe au microscope polarisant permettait, pour chaque valeur de la tension, de choisir la durée minimale d'impulsion provoquant le basculement complet du carré central (commande d'impulsion isolée, coup-par-coap).

A basse température, lorsque le basculement n'a plus lieu par propagation d'une paroi unique, la durée minimale d'impulsion provoquant le basculement complet ne permet plus de calculer une vitesse de propagation de paroi comparable à celle fournie par le premier procédé de mesure.

La figure 9 représente le logarithme de la vitesse de propagation de paroi en fonction de l'inverse du champ électrique, pour différentes températures, mesuré par le premier procédé sur un cristal d'épaisseur $d=58 \mu \mathrm{m}$. On trouve la même loi exponentielle que dans le cas du

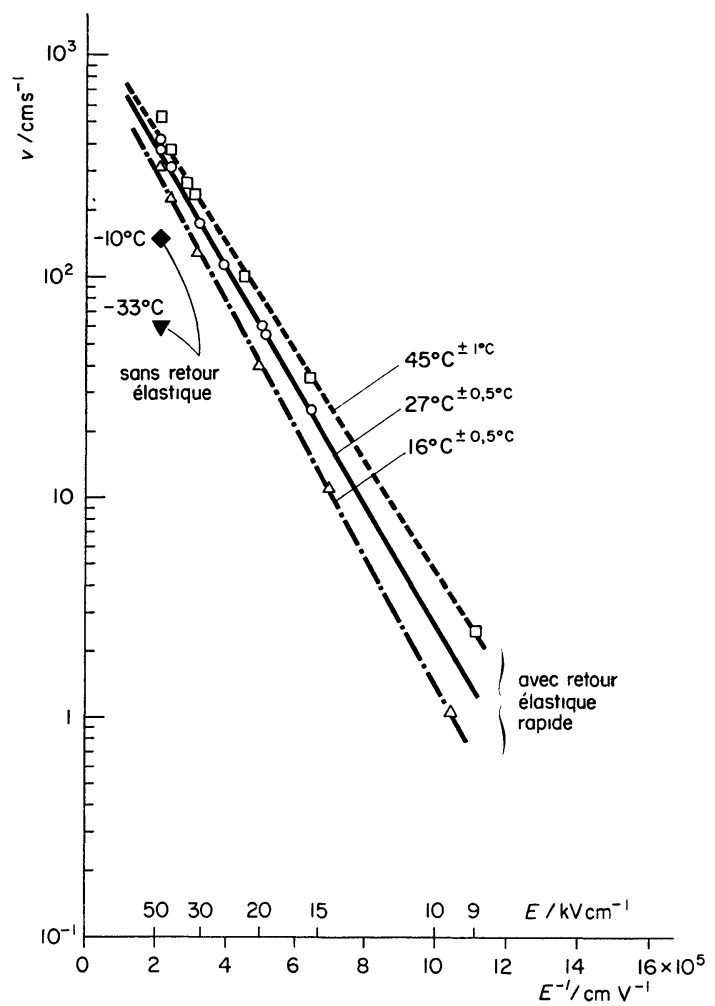

FIG. 9. - Variation de la vitesse de propagation de paroi (échelle logarithmique) en fonction de l'inverse du champ électrique, pour différentes températures (procédé $\mathrm{n}^{\circ} 1$, fenêtre de $190 \mu \mathrm{m}$ de diamètre, mesure photométrique, épaisseur de la lame : $58 \mu \mathrm{m})$.

cristal à épaisseur $d=96 \mu \mathrm{m}$ (Fig. 6), quoique avec des constantes $v_{\infty}$ et $\delta$ différentes. La figure 10 représente le logarithme de la vitesse de propagation de

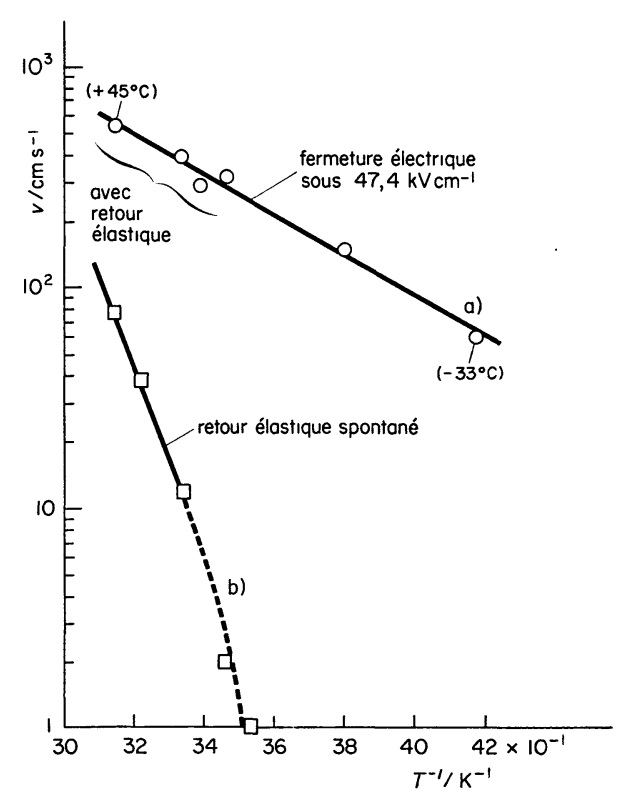

Fig. 10. - Variation de la vitesse de propagation de paroi (échelle logarithmique) 'en fonction de l'inverse de la température ; a) Fermeture électrique sous $47,4 \mathrm{kV} \mathrm{cm}^{-1}$; b) Retour élastique spontané ; épaisseur de la lame : $58 \mu \mathrm{m}$. L'effet de l'angle de cisaillement se traduit par l'augmentation beaucoup plus rapide de la vitesse de retour élastique lorsque la température monte. 
paroi en fonction de l'inverse de la température, à champ électrique $E=47,4 \mathrm{kV} \mathrm{cm}^{-1}$ d'une part, et pour le retour élastique spontané, d'autre part. La figure 11 représente l'inverse du temps de basculement

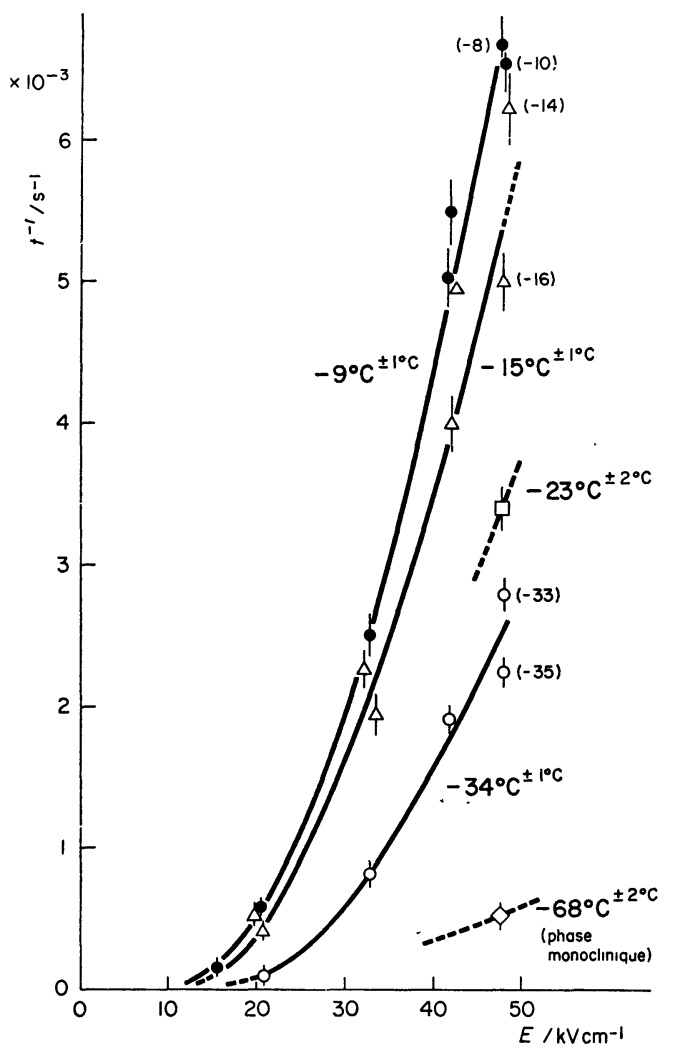

Fig. 11. - Variation de l'inverse du temps de basculement (proportionnel à une vitesse équivalente de paroi) du carré central d'une plaquette (photo $n^{\circ} 27$ ) à basses températurès en fonction du champ électrique (procédé $\mathrm{n}^{\circ} 2$, observation directe; $1 \times 10^{3} \mathrm{~s}^{-1}$ correspond à environ $0,22 \mathrm{~ms}^{-1}$ si le carré central bascule par mouvement symétrique de deux parois); épaisseur de la lame : $58 \mu \mathrm{m}$.

complet du carré central en fonction du champ électrique, pour différentes températures, mesuré par le second procédé ; la vitesse de paroi réelle ne peut pas en être déduite correctement. Le logarithme de l'inverse du temps de basculement à $47,4 \mathrm{kV} \mathrm{cm}^{-1}$ de ce cristal en fonction de l'inverse de la température est représenté sur la figure 12 .

On constate que la vitesse de propagation de paroi diminue de façon monotone lorsque la température s'abaisse. A $-81^{\circ} \mathrm{C}$ (phase trigonale !) le basculement est extrêmement lent ( $\sim 1 \mathrm{~min})$, même sous un champ de $54 \mathrm{kV} / \mathrm{cm}$. Aux températures encore plus basses, on n'observe plus aucun basculement : le seuil de basculement est supérieur à $54 \mathrm{kV} / \mathrm{cm}$.

5.9 MEsures AU-DesSUS DE LA TEMPÉRATURE AMBIANTE. - L'objectif des mesures à basse température était principalement de mettre en évidence les effets d'une diminution de l'angle de cisaillement. Or, l'abaissement de la température ne provoque pas seule-

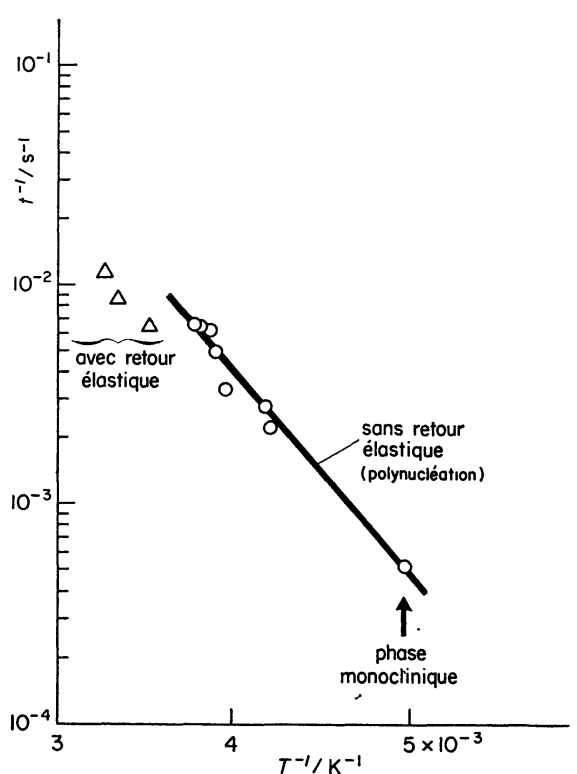

Fig. 12. - Variation de l'inverse du temps de basculement (échelle logarithmique) du carré central en fonction de l'inverse de la température ; champ électrique $E=47,4 \mathrm{kV} \mathrm{cm}^{-1}$; épaisseur du cristal : $53 \mu \mathrm{m}$

ment une diminution de l'angle de cisaillement, mais aussi de toute évidence une forte diminution de la mobilité de paroi, à tel point même que la nucléation de nouveaux domaines devient le mécanisme principal de basculement. La suppression du retour élastique aux températures inférieures à $10^{\circ} \mathrm{C}$ peut ainsi être interprétée au moins de deux façons :

a) L'angle de cisaillement ayant diminué, les contraintes mécaniques entre domaines antiparallèles ne suffisent plus à provoquer un mouvement de paroi ;

b) Le seuil de basculement a augmenté (comme le prouve la diminution de mobilité de paroi sous champ électrique) et les tensions mécaniques dues au cisaillement, étant inférieures au seuil mécanique, ne sont plus capables de provoquer le retour élastique.

Ces considérations ont justifié une série d'essais à température supérieure à l'ambiante. Des mesures sous flux d'azote chaud, à 34 et $46^{\circ} \mathrm{C}$, ont confirmé que la mobilité de paroi (sous champ électrique) augmente avec la température (Fig. 10). Mais la vitesse de paroi lors $\mathrm{du}$ retour élastique - quoique relativement petite - croît très vite en fonction de la température, comme le montre la figure 10 . Ce comportement est conforme à ce que l'on peut prévoir, à cause de l'augmentation de l'angle de cisaillement.

5.10 CONCLUSIONS TIRÉES DES MESURES DE BASCULEMENT AU-DESSOUS ET AU-DESSUS DE LA TEMPÉRATURE AMBIANTE. - L'ensemble de ces mesures permet de tirer les conclusions suivantes :

- Le retour élastique est freiné par un refroidissement, accéléré par un chauffage. 
- La mobilité de paroi décroît rapidement lorsque la température décroît.

- A température ambiante, la vitesse de paroi lors du retour élastique est beaucoup plus petite que la vitesse de paroi obtenue sous un champ électrique de 30 à $50 \mathrm{kV} / \mathrm{cm}$. Elle atteint au maximum $0,2 \mathrm{~m} / \mathrm{s}$, de l'ordre de la vitesse de paroi obtenue sous un champ électrique de $15 \mathrm{kV} / \mathrm{cm}$ (ce qui est proche du seuil effectif de basculement électrique).

- La dissymétrie de basculement en fonction de la polarité du champ électrique subsiste, et même se trouve accentuée à basse température.

- Pour obtenir les avantages attendus d'un angle de cisaillement faible, du point de vue de la vitesse de propagation de paroi, il faut chercher à

- diminuer cet angle par un autre moyen que l'abaissement de température (par exemple en développant des boracites mixtes à cisaillement nul),

- augmenter la mobilité de paroi (par exemple en améliorant la perfection des cristaux de boracites).

\section{Discussion. - 6.1 Comportement De LA VITESSE} DE PAROI, EN FONCTION DU CHAMP ÉLECTRIQUE. Les mesures de vitesse de paroi (à $180^{\circ}$ ) concernant la boracite $(\mathrm{Fe}, \mathrm{I})$, ont mis en évidence, pour le cas du cristal entièrement libre de contraintes extérieures et intérieures (absence d'autoblocage par domaines différemment orientés), une relation exponentielle entre la vitesse de paroi, $v$, et le champ électrique $E$ (à température constante) :

$$
v=v_{\infty} e^{-\delta / E} .
$$

La relation est valable au moins pour la plage de $E=0,7$ à $\sim 48 \mathrm{kV} \mathrm{cm}^{-1}$ et pour quatre ordres de grandeur de vitesse. Cela signifie qu'il n'y a pas de champ coercitif, propriété d'ailleurs commune à la plupart des ferro-électriques mécaniquement libres. La forme exponentielle est identique à celle qui a été trouvée par Miller et Savage $[25,26,27,28]$ pour le $\mathrm{BaTiO}_{3}$, par Itoh et ses collaborateurs [9] pour le $\mathrm{Pb}_{5} \mathrm{Ge}_{3} \mathrm{O}_{11}$ et par Hadni et coll. pour le TGS [31]. Lorsqu'un domaine basculé est partiellement ou totalement encadré par un domaine à polarisation opposée, on constate :

a) une relation approximativement linéaire entre champ électrique et vitesse de paroi au-dessus d'un champ seuil, et

b) un retour élastique (effet ressort) des domaines basculés.

Les mesures de vitesse de propagation de paroi font apparaître une très grande dissymétrie de vitesse en fonction de la polarité du champ appliqué. Le phénomène n'a pas encore trouvé une explication claire ; il n'est pourtant pas exclu que des tensions mécaniques résiduelles, provenant du processus de cristallisation, soient à son origine.

D'après Kumada [2] et Barkley [22], la vitesse de propagation de paroi dans le GMO est une fonction linéaire du champ électrique, au moins jusqu'à $8 \mathrm{kV} / \mathrm{cm}$ selon le premier auteur, et même jusqu'à $25 \mathrm{kV} / \mathrm{cm}$ selon le second. Il serait intéressant d'expliquer pourquoi le GMO ne se conforme pas à la loi de Miller et Savage comme le $\mathrm{BaTiO}_{3}$, le $\mathrm{Fe}_{3} \mathrm{~B}_{7} \mathrm{O}_{13} \mathrm{I}$ et le TGS [31].

6.2 EfFETS De Fatigue associés auX BasCULEMENTS ALTERNÉs. - Les essais de fatigue décrits aux chapitres 4.5.1 à 4.5.3 ont conduit à des résultats en partie contradictoires : dans certains cas, il n'y a pas de fatigue et les propriétés du cristal semblent plutôt s'améliorer (4.5.1 et 4.5.3); dans d'autres cas, au contraire, les propriétés du cristal se détériorent (4.5.2)

Cette contradiction n'est qu'apparente, parce que les plaquettes sur lesquelles les mesures ont été faites tombent dans deux catégories différentes de qualité (perfection cristalline et forme de plaquette). Lorsqu'une plaquette est d'assez bonne qualité pour qu'il soit possible de la polariser entièrement dans un sens (monodomaine), que sa forme est rectangulaire, ses côtés étant parallèles aux axes principaux du cristal, et qu'elle est entièrement métallisée sur ses deux grandes faces, on peut entreprendre des essais de fatigue au moyen de basculements complets. On constate alors une absence d'effets de fatigue, et une amélioration des paramètres de basculement (chapitres 4.5.1 et 4.5.3). Si l'on retient les hypothèses du chapitre précédent concernant les tensions mécaniques résiduelles, on peut voir dans cette amélioration une diminution des tensions internes, une symétrisation de ces tensions, ou encore une forme de relaxation du cristal favorisée par des basculements alternés complets.

Par contre, les essais conduisant à des effets de fatigue réelle concernent toujours des échantillons où le basculement n'est pas complet, ou bien parce que la qualité du cristal d'origine est insuffisante, ou bien parce que le champ de basculement n'est pas appliqué à toute la plaquette. Dans ce cas, la relaxation des tensions internes n'a pas lieu, et il semble même que le basculement partiel a plutôt tendance à faire augmenter ces tensions. Il se peut aussi que les tensions mécaniques initiales soient si élevées que, d'une part elles expliquent l'impossibilité de provoquer le basculement complet, et d'autre part elles engendrent souvent la rupture du cristal à plus ou moins brève échéance.

7. Conclusions. - On conclut que même l'angle de cisaillement très petit $(\sim 2 \mathrm{~min}$. à température ambiante) de la boracite ( $\mathrm{Fe}, \mathrm{I})$ ferro-électrique/ferroélastique d'espèce Aizu $\overline{4} 3 \mathrm{mFmm} 2$, peut encore provoquer - dans certaines conditions de géométrie - des interactions mécaniques entre domaines dans une plaque monocristalline monolithique.

L'utilisation de la boracite $(\mathrm{Fe}, \mathrm{I})$ en tant que pointmémoire (optique) nécessiterait donc, soit l'isolement mécanique de chaque élément, soit une structure de domaines en bandes parallèles, géométrie qui ne provoque pas de phénomènes de proximité mécaniques 
dans un cristal libre. L'absence d'un seuil de basculement, constatée au moins entre 0,7 et $48 \mathrm{kV} \mathrm{cm}{ }^{-1}$, ne gênerait pas pour la commande d'éléments isolés ou de domaines en bandes.

Afin d'éviter les effets mécaniques de proximité entre points-mémoire voisins dans une plaque cristalline, l'angle de cisaillement ne devrait pas être plus grand qu'une minute environ. Dans le cas d'une très grande mobilité de paroi de domaine, cette limite pourrait même être réduite à quelques secondes d'arc. Cette constatation est certainement tout aussi vraie pour tous les ferro-électriques/ferro-élastiques que l'on désirerait utiliser en tant que points-mémoire optique ou électrique (par exemple PLZT, GMO, $\mathrm{Bi}_{4} \mathrm{Ti}_{3} \mathrm{O}_{12}$ ). La diminution de l'angle de cisaillement est en principe possible pour certaines compositions de cristaux mixtes de boracites.

L'absence de fatigue ferro-électrique intrinsèque de la boracite $(\mathrm{Fe}, \mathrm{I})-10^{8}$ cycles sans endommagement pour un cristal mécaniquement libre - est un facteur extrêmement encourageant du point de vue de l'utilisation de ce matériau pour des mémoires ferro-électriques ou optiques. Tous les phénomènes de fatigue non intrinsèque observés pour des cristaux de boracite (Fe, I) peuvent en principe être évités grâce à l'emploi de technologies adéquates.
Les efforts futurs de la recherche devront surtout porter sur l'accroissement de la taille et sur l'amélioration de la qualité des cristaux, éventuellement sur des cristaux ou couches mixtes à cisaillement négligeable. Il y a pourtant lieu de souligner qu'une composition de boracite à cisaillement nul — de même d'ailleurs que tout autre ferro-électrique en l'absence de contrainte mécanique - sera caractérisée par l'absence d'un seuil vrai de basculement. L'adressage, d'un monocristal à cisaillement nul ou fini, ou d'une couche à cisaillement nul, exigera toujours un contact individuel pour chaque point-mémoire ou l'utilisation de composantes électroniques créant un seuil artificiel. Or, du point de vue de la fabrication, une composition à cisaillement nul faciliterait grandement la technologie en rendant superflue la séparation mécanique des éléments individuels d'une mémoire monolithique.

Remerciements. - Les auteurs remercient la Délégation à l'Informatique, à Paris, pour le soutien qu'elle a accordé à cette recherche. Ils tiennent également à remercier MM. G. Pircher et F. Micheron, ThomsonCSF, Corbeville, pour leur critique constructive tout au long du travail, et MM. H. Tippmann et K. Inderbitzin, de Battelle-Genève, pour leurs travaux très soignés de mesures diverses et de préparation d'échantillons.

\section{Bibliographie}

[1] Cummins, S. E. et LuKe, T. E., IEEE Trans. Eelctron Devices ED-18 (1971) 761-8.

[2] Kumada, A., IEEE Trans. Electron Devices ED-20 (1973) 866-73.

[3] Kumada, A., Phys. Lett. 30A (1969) 186-7.

[4] FunK, R. et Schmid, H., Brevet Suisse no 437.532 (1962).

[5] LaND, C. E., Metall. Trans. 2 (1971) 781-8.

[6] Land, C. E. et Thacher, P. D., Albers, W. A., Ed., Physics of Optoelectronic Materials (Plenum Publ. Corp., New York), 1971, p. 169-96.

[7] Iwasaki, H., SugiI, K., NiIzeki, N. et Toyoda, H., Ferroelectrics 3 (1972) 157-61.

[8] Iwasaki, H., Miyawa, S., Kolzumi, H., Suggil, K. et NiIzeKI, N., J. Appl. Phys. 43 (1973) 4907-15.

[9] IтоH, Y., Yamada, T., IwaSAKi, H. et MiYazawa, S., Digest of Technical Papers, Int. Conf. Solid State Devices, Tokyo, Aug. 29-31, 1973.

[10] Aizu, K., J. Phys. Soc. Japan 27 (1969) 387-96.

[11] Kobayashi, I., Mizutani, I., Hara, H., Yamada, N., NaKada, O., Kumada, A. et Schmid, H., J. Phys. Soc. Japan 28 (suppl.) (1970) 67-70.

[12] Kobayashi, J., Schmid, H. et Ascher, E., Phys. Stat. Sol. 26 (1968) 277-83.

[13] Kobayashi, J., Mizutani, I., Schmid, H. et Schachner, H., Phys. Rev. B 1 (1970) 3801-8.

[14] SCHMid, H., non publié.

[15] Brixner, L. H., Bierstedt, P. E., Sleight, A. W. et Licis, M. S., Mat. Res. Bull. 6 (1971) 545-54.
[16] Meitzler, A. H. et O’bryan Jr., H. M., Proc. IEEE 61 (1973) 959-974.

[17] Jona, F. et Shirane, G., Ferroelectric Crystals (Pergamon Press, Oxford) 1962, p. 126.

[18] Schmid, H., Chan, P., Petermann, L. A., Mändly, M. et Teufel, F., Ferroelectrics (en presse).

[19] Camlibel, I., J. Appl. Phys. 40 (1969) 1690.

[20] Pisarev, R. V., Druzhinin, V. V., Nesterova, N. N., Prochorova, S. D. et Andreeva, G. T., Phys. Stat. Sol. 40 (1970) 503-12.

[21] GaHM, J., Zeiss-Mitteilungen 3 (1964) 153-92.

[22] Barkley, J. R., Brixner, L. H., Hogan, E. M. et Waring Jr, R. K., Ferroelectrics 3 (1972) 191-7.

[23] Barkley, J. R. et Jeitschko, W., J. Appl. Phys. 44 (1973) 938-44.

[24] Вонм, J. et Kürsten, H. D., Phys. Stat. Sol. (a) 19 (1973) 179.

[25] Miller, R. C. et Savage, A., Phys. Rev. Lett. 2 (1959).

[26] Miller, R. C. et Savage, A., Phys. Rev. 115 (1959) 1176-80

[27] Miller, R. C. et Savage, A., J. Appl. Phys. 31 (1960) 662.

[28] Miller, R. C. et Weinreich, G., Phys. Rev. 117 (1960) 1460.

[29] Baldwin Jr, J. A. et Milstein, F., J. Appl. Phys. 45 (1974) 4006-12.

[30] Baldwin Jr, J. A. et Milstein F., J. Appl. Phys. 45 (1974) 4013-15.

[31] Hadni, A. et Thomas, R., C. R. Hebd. Séan. Acad. Sci (1975) à paraître. 